\title{
Diverse subgroup set discovery
}

\author{
Matthijs van Leeuwen • Arno Knobbe
}

Received: 31 October 2011 / Accepted: 21 May 2012 / Published online: 10 June 2012 (C) The Author(s) 2012. This article is published with open access at Springerlink.com

\begin{abstract}
Large data is challenging for most existing discovery algorithms, for several reasons. First of all, such data leads to enormous hypothesis spaces, making exhaustive search infeasible. Second, many variants of essentially the same pattern exist, due to (numeric) attributes of high cardinality, correlated attributes, and so on. This causes top- $k$ mining algorithms to return highly redundant result sets, while ignoring many potentially interesting results. These problems are particularly apparent with subgroup discovery (SD) and its generalisation, exceptional model mining. To address this, we introduce subgroup set discovery: one should not consider individual subgroups, but sets of subgroups. We consider three degrees of redundancy, and propose corresponding heuristic selection strategies in order to eliminate redundancy. By incorporating these (generic) subgroup selection methods in a beam search, the aim is to improve the balance between exploration and exploitation. The proposed algorithm, dubbed DSSD for diverse subgroup set discovery, is experimentally
\end{abstract}

Responsible editor: Dimitrios Gunopulos, Donato Malerba, Michalis Vazirgiannis.

The research described in this paper builds upon and extends the work appearing in ECML PKDD'11 (van Leeuwen and Knobbe 2011).

M. van Leeuwen $(\varangle)$

Machine Learning, Department of Computer Science, Katholieke Universiteit Leuven,

Leuven, Belgium

e-mail: matthijs.vanleeuwen@cs.kuleuven.be

M. van Leeuwen

Algorithmic Data Analysis, Department of Information and Computer Sciences, Faculty of Science, Universiteit Utrecht, Utrecht, The Netherlands

A. Knobbe

Leiden Institute of Advanced Computer Science, Universiteit Leiden, Leiden, The Netherlands e-mail: knobbe@liacs.nl 
evaluated and compared to existing approaches. For this, a variety of target types with corresponding datasets and quality measures is used. The subgroup sets that are discovered by the competing methods are evaluated primarily on the following three criteria: (1) diversity in the subgroup covers (exploration), (2) the maximum quality found (exploitation), and (3) runtime. The results show that DSSD outperforms each traditional SD method on all or a (non-empty) subset of these criteria, depending on the specific setting. The more complex the task, the larger the benefit of using our diverse heuristic search turns out to be.

Keywords Subgroup set discovery - Exceptional model mining · Pattern selection · Heuristic search · Diversity

\section{Introduction}

The field of subgroup discovery (SD) is concerned with the discovery of subsets of the data, where the target attribute(s) show an interesting difference in distribution, compared to that of the entire dataset. As such, the field encompasses all forms of discovery of local patterns in an exploratory, supervised setting. The typical definition of a SD task involves finding all subgroups that fit certain user-specified inductive constraints, and show a sufficiently high interestingness according to some chosen quality measure. SD algorithms have been developed for a large variety of data types, ranging from simple discrete attribute-value data, to very large and complex datasets of numeric and relational nature (see Sect. 8 for some examples). Especially in the case of these more challenging datasets, the traditional emphasis on completeness of the result is problematic.

In this paper, we address two important problems relating to the analysis of large and complex data. First, manual inspection of the resulting subgroup set is often hampered by the many subgroups reported and the high levels of redundancy in this result set. Second, when dealing with challenging data, for example when many high-cardinality attributes are involved, the hypothesis space becomes extremely large, and the whole discovery process becomes overly time-consuming. It turns out that both problems revolve around the notion of diversity in sets of subgroups. For the result set redundancy problem, achieving more diversity will clearly address the many very similar subgroups. For the large search space problem, we will argue that heuristic search becomes a necessity, and demonstrate that retaining diversity during the search process is of paramount importance.

In the majority of discovery algorithms, including those for SD (Klösgen 1996; Wrobel 1997), it is assumed that complete solutions to a particular discovery task are required, and thus some form of exhaustive search is employed. In order to obtain efficiency, these algorithms typically rely on top-down search combined with considerable pruning, exploiting either anti-monotonicity of the quality measure (e.g. frequency), or so-called optimistic estimates of the maximally attainable quality at every point in the search space (Grosskreutz et al 2008). With small datasets and simple tasks, these tricks work well and give complete solutions in reasonable time. However, on large and complex datasets, exhaustive approaches simply become infeasible, even 
when considerable pruning can be achieved. Additionally, we consider exceptional model mining (EMM) (Leman et al 2008; Duivesteijn et al 2010; van Leeuwen 2010), which allows multiple target attributes and complex models to be used for measuring quality. With EMM in particular, we are often dealing with quality measures that are not monotonic, and for which no optimistic estimates are available.

Apart from the computational concerns with discovery in large datasets, one also needs to consider the practicality of complete solutions in terms of the size of the output. Even when using condensed representations (Mannila and Toivonen 1996; Pasquier et al 1999) or some form of pattern set selection (Bringmann and Zimmermann 2007; Knobbe and Ho 2006b; Peng et al 2005) as a post-processing step, the end result may still be unrealistically large, and represent tiny details of the data overly specifically. The experienced user of discovery algorithms will recognise the large level of redundancy that is common in the final pattern set. This redundancy is often the result of dependencies between the (non-target) attributes, which lead to large numbers of variations of a particular finding. Note that large result sets are problematic even in top- $k$ approaches. Large result sets are obviously not a problem in top-1 approaches, but they are when $k \geq 2$, as the mentioned dependencies will lead to the top of the pattern list being populated with different variations on the same theme, and alternative patterns dropping out of the top- $k$. This problem is aptly illustrated by Fig. 1, which shows that the top-100 subgroups obtained on the Emotions dataset (see Sect. 7 on experiments) cover almost exactly the same tuples.

The obvious alternative to exhaustive search, and the one we consider in this paper, is heuristic search: employ educated guesses to consider only that fraction of the search space that is likely to contain the patterns of interest. When performing heuristic search, it is essential to achieve a good balance between exploitation and exploration. In other words, to focus and extend on promising areas in the search space, while leaving room for several alternative lines of search. In this work, we will implement this balance by means of beam search, which provides a good mixture between parallel search (exploration) and hill-climbing (exploitation). We will experiment with different variations of achieving diversity in the beam, that is, the current list of candidates to be extended. Due to the above-mentioned risk of redundancy with top- $k$ selection, the level of exploration within a beam can become limited, which will adversely affect the quality of the end result. Inspiration for selecting a diverse collection of patterns for the beam at each search level will come from pattern set selection techniques (Bringmann and Zimmermann 2007; Knobbe and Ho 2006b; Peng et al 2005), which were originally designed for post-processing the end-result of discovery algorithms.

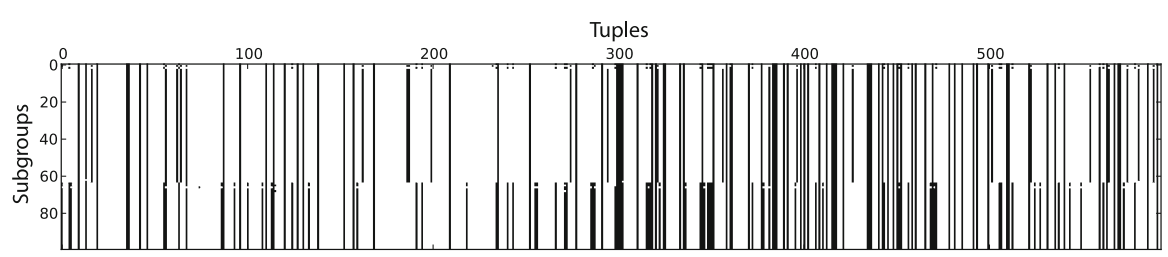

Fig. 1 Redundancy in top- $k$ EMM. Shown are the covers (in black) of the top-100 subgroups obtained using a beam search on Emotions with a quality measure based on the Kullback-Leibler divergence 
1.1 Redundancy in subgroup sets

To better appreciate the reasons behind redundancy in subgroups sets, consider an example from practice that relates to car accidents. Assume the car accidents are described by a number of attributes, including the following: ${ }^{1}$

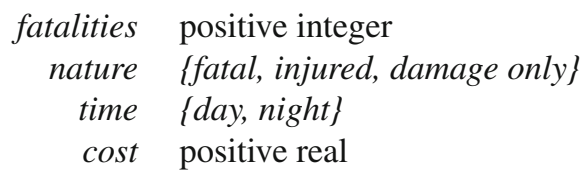

Although the attributes fatalities and nature are not perfectly correlated, they do convey some mutual information, and will lead to multiple similar patterns. This redundancy is already visible after a first analysis of depth-1 subgroups, where the following ranking of subgroups is obtained:

$$
\begin{array}{ll}
\text { 1. } & \text { fatalities } \geq 1 \\
\text { 2. } & \text { nature }=\text { fatal } \\
\text { 3. } & \text { fatalities } \geq 2 \\
\text { 4. } & \text { nature } \neq \text { damage only } \\
\text { 5. } & \text { time }=\text { night } \\
\text {.. } & \ldots
\end{array}
$$

Assuming a beam width $w=4$, only the first four subgroups will be used to build candidates for the next level, and as a result, the subgroup time $=$ night (and all lower ranking ones) will be ignored. On the next level, the ranking could be as follows:

1. fatalities $\geq 1 \wedge$ nature $=$ fatal

2. fatalities $\geq 1 \wedge$ nature $\neq$ damage only

3. fatalities $\geq 1 \wedge$ fatalities $\geq 2$

4. nature $=$ fatal $\wedge$ cost $\geq 123.4$

... $\ldots$

Note how the top of the ranking has become saturated with variations on the theme fatalities $\geq 1$. For example, the condition time $=$ night was not considered for the second level, due to the limited beam width, and any potentially interesting combinations with for example cost where never investigated.

Note that although our example suggests otherwise, redundancy in subgroup collections need not just be the result of strict functional dependencies in the data (such as fatalities $\geq 1 \rightarrow$ nature $=$ fatal $)$, but can also be due to dependencies of more statistical nature. As such, concepts such as condensed representations (Mannila and Toivonen 1996; Pasquier et al 1999) will not suffice to achieve diversity in the intermediate and final subgroups sets.

Such causes of redundancy, both in the search beam and the final result set, are particularly frequent in large and complex datasets. The most obvious form of complexity in datasets is large numbers of attributes. As the hypothesis space grows exponentially

\footnotetext{
1 The target attribute is not relevant here, but could for example convey whether the accident resulted in litigation.
} 
with the number of attributes, exhaustive search becomes prohibitively time-consuming. A further contributing factor in the size of the hypothesis space, one that is not relevant in for example frequent itemset mining, is the cardinality of attributes. As nominal attributes can assume more than two values, testing for equality will produce multiple possible refinements per candidate subgroup. The cardinality of an attribute is of particular relevance in the case of numeric attributes, as in theory, the cardinality of the attribute could be as high as the number of tuples in the dataset (e.g. think of the cost of a car accident). Although in our SD implementation, we do not test for every conceivable threshold in the continuous domain-we use a form of dynamic discretisation-numeric attributes are still a major factor in the run times. Finally, when the data in question no longer concerns attribute-value data, but more complex representations, such as relational (Knobbe 2006) or graphical (Yan and Han 2002), the search space becomes even larger.

\subsection{Contributions and roadmap}

This paper is a significant extension of our recent paper (van Leeuwen and Knobbe 2011) on non-redundant generalised subgroup discovery (GSD). Although the main message of the paper is unchanged, it encompasses important novel contributions. First of all, the beam selection methods are now presented as generic subgroup selection methods and three additional selection strategies that dynamically determine the number of subgroups to be selected are introduced. Second, the complete algorithm is separately introduced, explained and dubbed DSSD, which stands for diverse subgroup set discovery.

Third, the experiments now include a much wider range of datasets and comparisons to sequential and weighted covering methods, showing in more detail the benefits of our techniques on complex data. Apart from the previous binary and multitarget (EMM) settings, the experiments also include discovery with either nominal or numeric targets. These experiments demonstrate the use of diverse SD in a multi-class and regression setting, respectively. Additionally, the collection of quality measures considered has been extended, most notably for multi-class and continuous targets. Finally, throughout the paper, we have extended the descriptions in order to clarify details and provide more background, for example in the related work section.

In Sect. 2, we will first formalise both SD and EMM, after which we will recap the commonly used search techniques, including the standard beam search algorithm. Section 3 presents the quality measures that will be used in the experiments. We will then introduce the notion of subgroup set discovery in Sect. 4, and argue that it is better to mine subgroup sets rather than individual subgroups, to ensure diversity. This leads to the non-redundant GSD problem statement. We will show that redundancy in subgroup sets can be formalised in (at least) three different ways, each subsequent definition being stricter than its predecessor. Each of these three degrees of redundancy is used as principle for two subgroup selection strategies in Sect. 5. The complete DSSD algorithm is presented in Sect. 6, and includes a method for pruning individual subgroup descriptions and a detailed description of the refinement operator. After that, we continue with an extensive empirical evaluation in Sect. 7. We round up with related work and conclusions in Sects. 8 and 9. 


\section{Preliminaries}

\subsection{SD and EMM}

We assume that the tuples to be analysed are described by a set of attributes $A$, which consists of $k$ description attributes $D$ and $l$ model (or target) attributes $M$ ( $k \geq 1$ and $l \geq 1$ ). In other words, we assume a supervised setting, with at least a single target attribute $M_{1}$ (in the case of classical SD), but possibly multiple attributes $M_{1}, \ldots, M_{l}$ (in the case of EMM). Each attribute $D_{i}$ (resp. $M_{i}$ ) has a domain of possible values $\operatorname{Dom}\left(D_{i}\right)\left(\operatorname{resp} . \operatorname{Dom}\left(M_{i}\right)\right)$. Our dataset $\mathcal{S}$ is now a bag of tuples $t$ over the set of attributes $A=\left\{D_{1}, \ldots, D_{k}, M_{1}, \ldots, M_{l}\right\}$. We use $x^{D}$ resp. $x^{M}$ to denote the projection of $x$ onto its description resp. model attributes, e.g. $t^{D}=\pi_{D}(t)$ in case of a tuple, or $\mathcal{S}^{M}=\pi_{M}(\mathcal{S})$ in case of a bag of tuples. Equivalently for individual attributes, e.g. $\mathcal{S}^{M_{i}}=\pi_{M_{i}}(\mathcal{S})$.

Arguably the most important concept in this paper is the subgroup, which consists of a description and corresponding cover.

Definition 1 (Subgroup cover) A subgroup (cover) is a bag of tuples $G \subseteq \mathcal{S}$ and $|G|$ denotes its size, also called subgroup size or coverage.

Definition 2 (Subgroup description) A subgroup description is an indicator function $s$, as a function of description attributes $D$. That is, it is a function $s:\left(\operatorname{Dom}\left(D_{1}\right) \times\right.$ $\left.\ldots \times \operatorname{Dom}\left(D_{k}\right)\right) \mapsto\{0,1\}$, and its corresponding subgroup cover is $G_{s}=\{t \in \mathcal{S} \mid$ $\left.s\left(t^{D}\right)=1\right\}$.

As is usual, in this paper a subgroup description is a pattern, consisting of a conjunction of conditions on the description attributes, e.g. $D_{x}=$ true $\wedge D_{y} \leq 3.14$. Such a pattern implies an indicator function as just defined.

Given a subgroup $G$, we would like to know how interesting it is, looking only at its model (or target) data $G^{M}$. We quantify this with a quality measure.

Definition 3 (Quality measure) A quality measure is a function $\varphi: \mathcal{G}^{M} \mapsto \mathbb{R}$ that assigns a numeric value to a subgroup $G^{M} \subseteq \mathcal{S}^{M}$, with $\mathcal{G}^{M}$ the set of all possible subsets of $\mathcal{S}^{M}$.

$S D$ and EMM The above definitions allow us to define the two main variations of data mining tasks that feature in this paper: SD and EMM. As mentioned, in SD we consider datasets where only a single model attribute $M_{1}$ (the target) exists. We are interested in finding the top-ranking subgroups according to a quality measure $\varphi$ that determines the level of interestingness in terms of unusual distribution of the target attribute $M_{1}$ :

Problem 1 (Top- $k S D$ ) Suppose we are given a dataset $\mathcal{S}$ with $l=1$, a quality measure $\varphi$ and a number $k$. The task is to find the $k$ top-ranking subgroups $\mathcal{G}_{k}$ with respect to $\varphi$.

EMM is a generalisation of the well-known SD paradigm, where the single target attribute is replaced by a collection of model attributes (Leman et al 2008). Just like in 
SD, EMM is concerned with finding subgroups that show an unusual distribution of the model attributes. However, dependencies between these attributes may occur, and it is therefore desirable to consider the joint distribution over $M_{1}, \ldots, M_{l}$. For this reason, modelling over $G^{M}$ is employed to compute a value for $\varphi$. If the model induced on $G^{M}$ is substantially different from the model induced on $\mathcal{S}^{M}$, quality is high and we call this an exceptional model. We can now formally state the EMM problem.

Problem 2 (Top- $k$ EMM) Suppose we are given a dataset $\mathcal{S}$, a quality measure $\varphi$ and a number $k$. The task is to find the $k$ top-ranking subgroups $\mathcal{G}_{k}$ with respect to $\varphi$.

\subsection{Subgroup search}

To find high-quality subgroups, the usual choice is a top-down search strategy. The search space is traversed by starting with simple descriptions and refining these along the way, from general to specific. For this a refinement operator that specialises subgroup descriptions is needed. For example, given the empty subgroup description, the refinement operator generates all descriptions consisting of a single condition. Given any subgroup description $X$, consisting of $|X|$ conditions, it generates all allowed descriptions of size $|X+1|$ containing $X$. These are the refinements of $X$.

Algorithms for SD commonly use the following two parameters to restrict the search space. A minimum coverage threshold (mincov) is used to ensure that a subgroup covers at least a certain number of tuples. A maximum depth (maxdepth) parameter imposes a maximum on the number of conditions a description may contain. The term depth refers to the shape of the search space, which can be regarded a tree.

Exhaustive search When exhaustive search is possible, depth-first search is commonly used. This is often the case for moderately sized nominal datasets with a single (binary) target. Whenever possible, (anti-)monotone properties of the quality measure are used to prune parts of the search space, a technique that is also commonly used in frequent pattern mining (Han et al 2007).

When this is not possible, so-called optimistic estimates (Grosskreutz et al 2008) can be used to restrict the search space. An optimistic estimate function computes the highest possible quality that any refinement of a subgroup could give. If this upper bound is lower than the quality of the current $k$ th subgroup, the current branch of the search space can be safely skipped without affecting the outcome of the algorithm. Depending on $k$, the dataset and the quality measure, this may lead to significant speed-ups.

Beam search When exhaustive search is not feasible, beam search (Lowerre 1976) is the widely accepted heuristic alternative. It is similar to exhaustive approaches in that it also uses a top-down strategy and a refinement operator, but it explores only part of the search space. For this, a level-wise search is performed, as bread-first search would do. However, on each level only a selection of all evaluated subgroups, i.e. the beam, is used for refinement.

On each level a refinement operator generates subgroups for the next level from each individual subgroup in the beam. The initial candidate set is generated from the empty subgroup description. From all generated candidates on a particular level, the $w$ highest ranking candidates (with respect to quality measure $\varphi$ ) are selected as beam. 
During the search, a final result list is maintained, in which the overall top- $k$ of all evaluated subgroups are kept.

Covering schemes To obtain diversity in rule induction and $\mathrm{SD}$, so-called 'covering' schemes have been introduced. The well-known rule induction algorithm CN2 (Clark and Niblett 1989; Clark and Boswell 1991) introduced what we will call sequential covering. Each round, the search procedure looks for the best rule, and subsequently all training examples covered by this rule are removed. This procedure is repeated until a stopping criterion is met (e.g. all examples are covered). This way, an ordered rule set is induced. The authors also proposed a slightly different variant that generates an unordered rule set, but we do not consider that here because it can only be applied when there is a single nominal class label.

Inspired by CN2, Lavrač et al (2004) introduced an adaptation of CN2 for SD, aptly named CN2-SD. One of the modifications they proposed is a new covering scheme, to reflect the different goals of SD: in contrast to rule induction, it does not aim to maximise accuracy. Rather, it is meant to be exploratory and aims to give an overview of regions of the data that stand out with respect to the target. For this, it should be possible for subgroups to overlap, which is not possible with sequential covering.

To achieve this, weighted covering was proposed. Instead of completely removing all tuples in a subgroup cover, all tuples in the database with a positive class label are assigned a weight. All tuples start with a weight of 1, but when a tuple is covered its weight decreases. Furthermore, the weighted relative accuracy (WRAcc) quality measure is adapted to take these weights into account. For a tuple $t$ that has been covered $i$ times, its weight $w(t, i)$ can be computed in two ways. With multiplicative weights: $w(t, i)=\gamma^{i}$ (for a given parameter $0<\gamma<1$ ), or with additive weights: $w(t, i)=\frac{1}{i+1}$.

We will empirically compare our methods to sequential covering and (a more generic variation on) multiplicative weighted covering in Sect. 7.

Exception maximisation description minimisation (EMDM) In van Leeuwen (2010) we introduced an alternative heuristic algorithm for EMM called EMDM. It is based on the observation that in EMM, both the description and the model data can be exploited to guide the search. Starting from a set of candidates that are likely to be of reasonable quality, subgroups are improved in an iterative manner. The first step, called exception maximisation, modifies the cover of a subgroup such that its quality is maximised. Next, the second step, called description minimisation, modifies the subgroup such that it can be succinctly described. These steps are alternately performed until a stable solution is found.

\section{Quality measures}

In this section we present the quality measures for individual subgroups that we will use in our experiments. Table 1 shows with which considered target types the quality measures can be used.

WRAcc WRAcc (Lavrač et al 2004) is a well-known SD quality measure for datasets with a single binary target attribute. It consists of two components: (1) the (relative) size of the subgroup and (2) the (relative) amount of positive examples that the 
Table 1 Quality measures can only be used in combination with certain target types

\begin{tabular}{|c|c|c|c|c|c|c|c|}
\hline Target type & WRAcc & MWRAcc & NWRAcc & $\chi^{2}$ & MT & WKL & WKG \\
\hline Single binary & $\checkmark$ & & & & & $\checkmark$ & \\
\hline Single nominal & & $\checkmark$ & & $\checkmark$ & & $\checkmark$ & \\
\hline Single numeric & & & $\checkmark$ & & $\checkmark$ & & \\
\hline Multiple binary & & & & & & $\checkmark$ & $\checkmark$ \\
\hline Multiple nominal & & & & & & $\checkmark$ & \\
\hline
\end{tabular}

All possible combinations are indicated with a checkmark

subgroup contains. Both of these positively contribute to a higher WRAcc. Let $1^{G}$ $\left(\right.$ resp. $1^{\mathcal{S}}$ ) denote the fraction of ones in the target attribute, within the subgroup (resp. entire dataset). The measure is then defined as

$$
\varphi_{W R A c c}(G)=\frac{|G|}{|\mathcal{S}|}\left(1^{G}-1^{\mathcal{S}}\right) .
$$

Multi-class weighted relative accuracy (MWRAcc) Abudawood and Flach (2009) introduced several multi-class versions of WRAcc. We here adopt one, namely the one-vs-rest variant, because the experiments in Abudawood and Flach (2009) show that the differences between the different versions are marginal.

The principle of the one-vs-rest MWRAcc is simple: one can apply the regular 'one-vs-one' WRAcc measure by setting one of the target values to 'positive' and the rest to 'negative'. By doing this procedure once for each possible target value and summing the qualities obtained this way, an overall quality can be computed. That is, the measure can be defined as

$$
\varphi_{M W R A c c}(G)=\sum_{x \in \operatorname{Dom}\left(M_{1}\right)}\left|W R A c c_{x}(G)\right|,
$$

where $W R A c c_{x}(G)$ means that $\varphi_{W R A c c}(G)$ is computed with $x$ as positive class.

Numeric weighted relative accuracy (NWRAcc) NWRAcc is a straightforward translation of regular WRAcc to the numeric case. Let $\mu^{G}$ (resp. $\mu^{\mathcal{S}}$ ) denote the mean of all target values in the subgroup (resp. the entire dataset). The measure is then defined as

$$
\varphi_{\text {WRAcc }}(G)=\frac{|G|}{|\mathcal{S}|}\left(\mu^{G}-\mu^{\mathcal{S}}\right)
$$

Chi-squared $\left(\chi^{2}\right)$ The $\chi^{2}$ test determines whether subgroup and class membership of a random tuple are statistically independent. Under the null hypothesis of assuming independence of columns and rows, the expected class frequencies can be computed from the marginals. Let $x^{G}$ (resp. $x^{\mathcal{S}}$ ) denote the fraction of tuples within the subgroup (resp. entire dataset) that have $x$ as target value, for $x \in \operatorname{Dom}\left(M_{1}\right)$. Then, for a subgroup $G$ the expected frequency for a class $x$ is $\frac{|G|}{|\mathcal{S}|} x^{\mathcal{S}}$. The $\chi^{2}$ statistic is the sum 
of the squared differences between observed and expected frequencies divided by the expected frequencies, and can be written as

$$
\varphi_{\chi^{2}}(G)=\sum_{x \in \operatorname{Dom}\left(M_{1}\right)}\left(\frac{\left[|G|\left(x^{G}-x^{\mathcal{S}}\right)\right]^{2}}{|G| x^{\mathcal{S}}}+\frac{\left[|G| x^{G}-|G| x^{\mathcal{S}}\right]^{2}}{(|\mathcal{S}|-|G|) x^{\mathcal{S}}}\right)
$$

Mean test (MT) The MT was already introduced in the Explora system (Klösgen 1996). It quantifies the difference between the means of the target value in the subgroup and in the complete dataset and is defined as

$$
\varphi_{M T}(G)=\sqrt{|G|}\left(\mu^{G}-\mu^{\mathcal{S}}\right),
$$

where $\mu^{G}$ and $\mu^{\mathcal{S}}$ are defined as before. Note that subgroups with a high MT are relatively large subgroups with a relatively high mean. Subgroups with a mean that is very low compared to the overall distribution have a negative quality; the absolute value of the difference between the means could be taken if one is also interested in those subgroups.

Weighted Kullback-Leibler divergence (WKL) The KL divergence (Kullback and Leibler 1951) is an asymmetric measure of the difference between probability distributions $P$ and $Q$. It quantifies the number of extra bits which would be required to encode a sample from $P$ using a code based on $Q$ ('wrong' distribution) instead of using a code based on $P$ ('correct' distribution).

For probability distributions $P$ and $Q$ of a discrete random variable, the KL divergence of $Q$ from $P$ is given as

$$
\mathrm{KL}(P \| Q)=\sum_{x} P(x) \log _{2} \frac{P(x)}{Q(x)} .
$$

We previously introduced a measure based on the KL divergence in van Leeuwen (2010). We assume that each attribute-value in our database is an independently drawn sample from an underlying, independent discrete random variable, and empirically estimate the probability distribution for each attribute $M_{i}$. We denote the function which derives such an empirical distribution by $\hat{P}$, which is defined as $\hat{P}\left(M_{i}=x\right)=$ $\frac{\left|\left\{t \in \mathcal{S} \mid t^{M^{i}}=x\right\}\right|}{|\mathcal{S}|}$. We then defined KL exceptionality as the sum of KL divergences over all individual attributes, from subgroup to database.

We here present an alternative that weighs quality by subgroup size, because this works better in combination with a level-wise search (without this weight, smaller subgroups tend to be of higher qualities). It is quite versatile as it can be easily used with a single or multiple binary model attributes, but also with nominal attributes.

Definition 4 (WKL quality) Given a database $\mathcal{S}$ and subgroup $G$, define (independent) Weighted KL quality as

$$
\varphi_{\mathrm{WKL}}\left(G^{M}\right)=\frac{|G|}{|\mathcal{S}|} \sum_{i=1}^{l} \operatorname{KL}\left(\hat{P}\left(G^{M_{i}}\right) \| \hat{P}\left(\mathcal{S}^{M_{i}}\right)\right)
$$


WKL quality has the potential advantage that it treats all values equally; unlike many quality measures for SD, $1 \mathrm{~s}$ and 0 s are considered symmetrically. When one is interested in deviating distributions, this is generally a desirable property. A downside of this quality measure is that all attributes are assumed to be completely independent. This assumption is likely to be violated when there are correlations between the model attributes.

Weighted Krimp gain (WKG) In van Leeuwen (2010) we introduced a second measure that, contrary to (Weighted) KL quality, does take associations between attributes into account. Another important difference is that it is asymmetric: it only considers $1 \mathrm{~s}$ and neglects the 0 s in the model data. Finally, it only works for binary data, although the generic idea (i.e. using compression to quantify differences between overall and subgroup distributions) could be applied to other types of data.

The WKG measure uses KRIMP code tables (Vreeken et al 2011) as models. These are ordered lists of itemsets that have codes associated to them. A code table can be used to encode a binary database by replacing each occurrence of an itemset with its associated code. KRIMP is a heuristic that approximates the optimal code table for a given database.

The basic principle is equivalent to that of WKL: a subgroup is interesting if it can be compressed (much) better by its own compressor, than by the compressor induced on the overall database. Similar to $W K L$ quality, we here introduce a weighted alternative to take the size of the subgroup into account.

Definition 5 (WKG) Let $\mathcal{D}$ be a binary database, $G \subseteq \mathcal{D}$ a subgroup, and $C T_{\mathcal{D}}$ and $C T_{G}$ their respective optimal code tables. We define the $W K G$ of group $G$ from $\mathcal{D}$, denoted by $\operatorname{WKG}(G \| \mathcal{D})$, as

$$
\mathrm{WKG}(G \| \mathcal{D})=L\left(G \mid C T_{\mathcal{D}}\right)-L\left(G \mid C T_{G}\right)
$$

with $L(G \mid C T)$ the size of $G$, in bits, encoded with code table $C T$.

Given this, defining the quality measure is straightforward.

Definition 6 (WKG quality) Let $\mathcal{S}$ be a database and $G \subseteq \mathcal{S}$ a subgroup. Define Weighted $K G$ quality as

$$
\varphi_{\mathrm{WKG}}\left(G^{M}\right)=\mathrm{WKG}\left(G^{M} \| \mathcal{S}^{M}\right)
$$

\section{Non-redundant GSD}

As described in the introduction, redundancy can be a severe problem in discovery tasks such as SD and EMM. Many (slightly) different subgroup descriptions imply many (almost) equal subgroup covers that have (almost) equal similarity. This adversely affects the results of any search that aims to be complete, and top- $k$ search in particular. That is, the top- $k$ is likely to contain many variations of the same theme, all of high quality, while clearly different subgroups are not presented to the user at all.

This redundancy problem teaches us the following two important lessons. 
1. SD algorithms should never return the complete set of subgroups, but a condensed representation or 'interesting' selection thereof.

2. Subgroups should not be considered only individually, they should always be judged also on their joint merit.

That is, we should consider subgroup set discovery: discovery of a non-redundant set of high-quality subgroups. This aim is comparable to recent pattern set selection approaches (Bringmann and Zimmermann 2007; Knobbe and Ho 2006b; Peng et al 2005), although we focus on SD and EMM here. The revised task can be formulated as follows.

Problem 3 (Non-redundant GSD) Suppose we are given a dataset $\mathcal{S}$, a quality measure $\varphi$ and a number $k$. The task is to find a non-redundant set $\mathcal{G}$ of $k$ high-quality subgroups.

The term GSD is used to emphasise that it encompasses both SD (single target) and EMM (multiple targets).

Given this task, the primary challenge is to define redundancy. Although it may be clear to a domain expert or data miner whether a (small) set of patterns contains redundancy or not, formalising redundancy is no trivial task. Several different approaches to formalising non-redundant pattern sets exist, e.g. using joint entropy (Cover and Thomas 2006; Knobbe and Ho 2006a; Bringmann and Zimmermann 2007), or minimum description length (MDL) (Grünwald 2007; Vreeken et al 2011). Sequential and weighted covering, as described in Sect. 2, also aim to increase diversity in the resulting subgroups.

However, very few of the existing methods can be straightforwardly applied to the task just mentioned, as they all make more specific assumptions about the task. For example, many methods assume that all description attributes are binary, and/or that there is a single binary target. We aim to develop methods that are specifically tailored to the GSD setting, without making any additional assumptions; they should work for any SD or EMM setting.

We consider three degrees of attaining diversity, which we consider equivalent to removing redundancy. The definitions in Sect. 2 show that a subgroup consists of a description and corresponding cover, which can be regarded as a subgroup's intent resp. extent. This suggests that redundancy can be defined both intensionally and extensionally. Further, in the EMM setting, we can also avail the models that are fitted on the subgroups, which suggests a third way to define redundancy. Hence, in a nonredundant subgroup set $\mathcal{G}$, all pairs $G_{i}, G_{j} \in \mathcal{G}$ (with $i \neq j$ ) should have substantially different:

1. subgroup descriptions, or

2. subgroup covers, or

3. exceptional models. (Only in the case of EMM.)

Note that each subsequent degree is stricter than its predecessor. On the first, least restrictive degree, substantially different descriptions are allowed, ignoring any potential similarity in the covers. The second degree of redundancy would also address similarity in the subgroup covers. The third degree of redundancy will consider subgroups 
that are different in both description and cover, and will address their difference in terms of the associated models built on the model attributes $M$. As these models will typically somehow represent the data distribution of the model data, this definition of redundancy implicitly enforces subgroups to have different data distributions.

Without being subjective, it is impossible to state that one of these definitions of redundancy is absolutely better than the others. This depends much on the data and the requirements of the data miner or domain expert. Hence we do not choose one of the degrees, but proceed considering each of them. In Sect. 5, each of the three degrees will be used as basic principle for two subgroup set selection methods.

\subsection{Quantifying redundancy in subgroup covers}

To be able to judge the effect of our methods and compare it to existing methods, such as the beam search described in Sect. 2, it is imperative that we quantify redundancy. Here, we focus on measuring redundancy in the subgroup covers, i.e. it matches best with the second degree of redundancy.

In the work this paper builds upon (van Leeuwen and Knobbe 2011), we introduced a measure to quantify redundancy in the covers of subgroup sets. For this measure, dubbed cover redundancy (CR), we assume that a maximally diverse set of subgroups would uniformly cover all tuples in the dataset. Also, let the cover count of a tuple be the number of times it is covered by a subgroup in a subgroup set. Given this assumption and definition, one can easily compute an expected cover count and measure how far each individual tuple's cover count deviates from this. This results in the following definition:

Definition $7(C R)$ Suppose we are given a dataset $\mathcal{S}$ and a set of subgroups $\mathcal{G}$. Define the cover count of a tuple $t \in \mathcal{S}$ as $c(t, \mathcal{G})=\sum_{G \in \mathcal{G}} s_{G}(t)$. The expected cover count $\hat{c}$ of a random tuple $t \in \mathcal{S}$ is defined as $\hat{c}=\frac{1}{|\mathcal{S}|} \sum_{t \in \mathcal{S}} c(t, \mathcal{G})$. Cover redundancy CR is now computed as:

$$
\mathrm{CR}^{\mathcal{S}}(\mathcal{G})=\frac{1}{|\mathcal{S}|} \sum_{t \in \mathcal{S}} \frac{|c(t, \mathcal{G})-\hat{c}|}{\hat{c}}
$$

The larger the CR is, the larger is the deviation from the uniform cover distribution. Because GSD aims to find only those parts of the data that stand out, this measure on itself does not tell us much; we cannot expect all tuples to be uniformly covered. However, CR is very useful when comparing different subgroup sets on the same dataset. If we have several subgroup sets of (roughly) the same size and for the same dataset, a lower CR indicates that fewer tuples are covered by more subgroups than expected, and thus the subgroup set is more diverse/less redundant.

In recent work on pattern set selection (Knobbe and Ho 2006a,b; Bringmann and Zimmermann 2007; Knobbe and Valkonet 2009), alternative measures for diversity in pattern sets were investigated. The most prominent of these, joint entropy, involves the information theoretical concept of entropy (Cover and Thomas 2006) over the binary features defined by each pattern (or subgroup, for that matter). 
Definition 8 (Joint entropy) Suppose that $\mathcal{G}=\left\{G_{1}, \ldots, G_{k}\right\}$ is a set of subgroups, and $B=\left(b_{1}, \ldots, b_{k}\right) \in\{0,1\}^{k}$ is a tuple of binary values. Let $p\left(s_{G_{1}}=b_{1}, \ldots, s_{G_{k}}=b_{k}\right)$ denote the fraction of tuples $t \in \mathcal{S}$ such that $s_{G_{1}}(t)=b_{1} \wedge \ldots \wedge s_{G_{k}}(t)=b_{k}$. The joint entropy of $\mathcal{G}$ is defined as:

$$
H(\mathcal{G})=-\sum_{B \in\{0,1\}^{k}} p\left(s_{G_{1}}=b_{1}, \ldots, s_{G_{k}}=b_{k}\right) \log _{2} p\left(s_{G_{1}}=b_{1}, \ldots, s_{G_{k}}=b_{k}\right)
$$

Note that $H$ is measured in bits, and each subgroup provides at most 1 bit of information, such that $H(\mathcal{G}) \leq|\mathcal{G}|$. Equality only occurs in the unlikely event that all subgroups cover half of the dataset, and each pair of subgroup covers is independent. Joint entropy has an inverse interpretation from CR: a high joint entropy between subgroups indicates low redundancy.

Informal comparison between the two measures shows that the measures are sometimes highly correlated (although inverted), and sometimes seem completely unrelated. This is unsurprising, as CR is computed from the cover count (the number of times a tuple is covered), whereas the joint entropy focuses on the counts of tuples with identical covers.

Example 1 As an example, the subgroup set visualised in Fig. 1 has a high CR of 1.43. Its joint entropy equals 1.573 bits, which indicates that not more information is conveyed with these 100 subgroups than could be conveyed with just 2 subgroups (which would represent 2 bits in the ideal case). Clearly, this cover distribution is highly undesirable, and much lower values for $\mathrm{CR}$ and much higher values for $\mathrm{H}$ are preferred.

\section{Diverse subgroup set selection}

In this section, we show how the three degrees of redundancy that we identified previously can be translated to subgroup selection strategies: procedures that select a small set of high-quality subgroups from a large number of candidate subgroups. The most naive strategy is to always select the $k$ top ranking subgroups with respect to quality; this is the default used by the SD beam search outlined in Sect. 2.2 and we will refer to this as the TopK strategy.

Since we intend to incorporate the selection strategies within level-wise search, it is important that they are computationally not too heavy. Unfortunately, most pattern set selection criteria require considering all possible pattern sets to ensure that the global optimum is found. Because we will need to select subgroup sets from large numbers of subgroups multiple times in a single run, such exhaustive strategies are infeasible. Hence, we resort to greedy and heuristic methods, as is usual in pattern set selection (Bringmann and Zimmermann 2007; Knobbe and Ho 2006b; Peng et al 2005).

In the following, a pair of strategies will be introduced for each of the three degrees of redundancy, where one selects a fixed number $k$ of subgroups and the other selects a variable number of subgroups. Each subsequent degree of redundancy is stricter than its predecessor, but also results in computationally more demanding procedures. This 
offers the data miner the opportunity to trade-off diversity with computation time. The aim of the variable-size strategies is to select fewer subgroups than their fixed-size counterparts, providing fast alternatives that still give reasonable results.

\subsection{Description-based subgroup selection}

Here we select subgroups purely on the basis of their descriptions, not considering their corresponding covers whatsoever. This is fast and potentially eliminates quite some redundant subgroups.

Fixed-size description-based selection $(\operatorname{Desc}(k))$ This strategy greedily selects subgroups by comparing each candidate to the subgroups already selected. If there is a selected subgroup that has (1) equal quality and (2) the same conditions except for one, the candidate is skipped.

The procedure to achieve this is as follows. Order all candidate subgroups descending on quality and consider them one by one until the desired number of subgroups $k$ is reached. For each considered subgroup $G \in$ Cands, discard it if its quality and all but one conditions are equal to that of any $G \in S e l$, otherwise include it in the selection. Time complexity for selecting a subgroup set is $\mathcal{O}(|\operatorname{Cands}| \cdot \log (|\operatorname{Cand}|)+$ $\mid$ Cand $s \mid \cdot$ maxlen) (where maxlen is the maximum number of conditions any description contains).

Variable-size description-based selection $(\operatorname{VarDesc}(c, l))$ An alternative way to achieve diversity is to allow each description attribute to occur only $c$ times in a condition in a subgroup set. Because the number of occurrences of an attribute depends on the number of conditions per description, each attribute is allowed to occur $\mathrm{cl}$ times, where $l$ is the (maximum) length of the descriptions in the candidate set. The beam width now depends on the number of description attributes $|D|, c$ and $l$. This effectively results in a (more or less) static beam width per experiment.

Order all candidate subgroups descending on quality and consider them one by one. For each considered subgroup $G \in C$ ands, check whether any of its conditions specifies an attribute that has already been used $c l$ times. If so, discard the candidate, otherwise add it to selection $\mathrm{Sel}$ and update the attribute usage counts. Stop when all attributes occur $\mathrm{cl}$ times in the selection or when no more candidates are available. Time complexity for subgroup selection is $\mathcal{O}(\mid$ Cands $\mid \cdot \log (\mid$ Cands $\mid))$.

\subsection{Cover-based subgroup selection}

Taking subgroup covers into account is computationally more intensive than considering only the descriptions, but also results in more diversity.

Fixed-size cover-based selection (Cover $(k)$ ) A score based on multiplicative weighted covering (Lavrač et al 2004) is used to weigh the quality of each subgroup, aiming to minimise the overlap between the selected subgroups. This score is defined as

$$
\Omega(G, S e l)=\frac{1}{|G|} \sum_{t \in G} \alpha^{c(t, S e l)}
$$


where $\alpha \in\langle 0,1]$ is the weight parameter. The less often tuples in subgroup $G$ are already covered by subgroups in the selection, the larger the score. If the cover contains only previously uncovered tuples, $\Omega(G, S e l)=1$.

In $k$ iterations, $k$ subgroups are selected. In each iteration, the subgroup that maximises $\Omega(G, S e l) \cdot \varphi(G)$ is selected. The first selected subgroup is always the one with the highest quality, since the selection is empty and $\Omega(G, \mathrm{Sel})=1$ for all $G$. After that, the $\Omega$-scores for the remaining $C$ ands are updated each iteration. Complexity is $\mathcal{O}(k \cdot \mid$ Cands $|\cdot| \mathcal{S} \mid)$.

Variable-size cover-based selection (VarCover $(f)$ ) This selection procedure is equivalent to the fixed-size version, except for the stopping criterion. Subgroups are iteratively selected until no candidate subgroup meets the minimum score $\delta$ specified by parameter $f$. The minimum score is defined as a fraction of the quality of the top-ranking candidate, i.e. $\delta=f \cdot \max _{G \in C a n d s} \varphi(G)$. Selection stops when there is no $G \in C$ ands for which $\Omega(G, \mathrm{Sel}) \cdot \varphi(G) \geq \delta$.

\subsection{Compression-based beam selection}

To be able to do model-based beam selection, a (dis)similarity measure on models is required. For this purpose, we focus on the models used by the WKL and WKG quality measures. These measures have in common that they rely on compression; they assume a coding scheme and the induced models can therefore be regarded as compressors.

In case of $W K G$, the compressor is the code table induced by KRIMP. In case of $W K L$, the compressor replaces each attribute-value $x$ by a code of optimal length $L(x)$ based on its marginal probability, i.e.

$$
L(x)=-\log _{2}\left(\hat{P}\left(M_{i}=x\right)\right) .
$$

Adopting the MDL principle (Grünwald 2007), we argue that the best set of compressors is that set that together compresses the dataset best. Selecting a set of compressors is equivalent to selecting a set of subgroups, since each subgroup has exactly one corresponding compressor. Since exhaustive search is infeasible, we propose the following heuristic.

1. We start with the 'base' compressor that is induced on the entire dataset, denoted $C^{S}$. Each $t \in S$ is compressed with this compressor, resulting in encoded size $L\left(S \mid C^{S}\right)$.

2. Next, we iteratively search for the subgroup that improves overall compression most, relative to the compression provided by the subgroups already selected. That is, the first selected subgroup is always the top-ranked one, since its compressor $C^{1}$ gives the largest gain with respect to $L\left(S \mid C^{S}\right)$.

3. Each transaction is compressed by the last picked subgroup that covers it, and by $C^{S}$ if it is not yet covered by any. So, after the first round, part of the transactions are encoded by $C^{S}$, others by $C^{1}$.

4. Assuming this encoding scheme, select that subgroup $G \in \operatorname{Cands} \backslash\left\{C^{1}, \ldots\right\}$ that maximises $L\left(S \mid C^{S}, C^{1}, \ldots\right)-L\left(S \mid C^{S}, C^{1}, \ldots, G\right)$. 
5. Repeat step 4 until some stopping criterion is reached.

To perform this selection strategy, all compressors belonging to the subgroups of a certain level are required. If these can be kept in memory, the complexity of the selection scheme is $\mathcal{O}(k \cdot \mid C$ and $s|\cdot| \mathcal{S}|\cdot| M \mid)$, where $k$ is the (maximum) number of subgroups to be selected and $|M|$ is the number of model attributes. However, keeping all compressors in memory may not be possible. They could then be either cached on disk or reconstructed on demand, but both approaches severely impact runtimes.

Fixed-size compression-based selection (Compress $(k)$ ) Using the heuristic outlined above, a fixed-size selection scheme is obtained by choosing an appropriate stopping criterion: stop when the selection contains $k$ subgroups.

Variable-size compression-based selection (VarCompress) MDL provides us a natural and parameter-free stopping criterion for the variable-size scheme. That is, we should stop when compression cannot be improved, meaning that we should stop when $L\left(S \mid C^{S}, C^{1}, \ldots\right)-L\left(S \mid C^{S}, C^{1}, \ldots, G\right) \leq 0$.

\section{DSSD: diverse beam search for non-redundant discovery}

In this section we present the complete DSSD algorithm.

\subsection{The DSSD algorithm}

The six generic subgroup set selection strategies presented in the previous section were primarily designed to improve the standard SD beam search. Instead of simply choosing the - potentially highly redundant— top- $k$ subgroups for the beam, one of the six more advanced selection strategies is used.

The overall subgroup set discovery algorithm we propose, shown in Algorithm 1, consists of three phases. First, a beam search is performed to mine $j$ subgroups (lines 1-12), using any of the proposed subgroup selection strategies to select the beam on each level (10). The refinement operator used on line 5 is described in more detail in the next subsection. In the second phase, each of the $j$ resulting subgroups is individually improved using dominance pruning (see Sect. 6.3), and syntactically equivalent subgroups are removed (13-16). As the final result set potentially still suffers from the redundancy problems of top- $k$-selection (line 8), in the third phase subgroup selection is used to select $k$ subgroups $(k \ll j)$ from the remaining subgroups. For this, the same strategy as during the beam search is used.

The DSSD algorithm has the following parameters. Dataset $\mathcal{S}$ and quality measure $\varphi$ are probably the most important parameters. $j$ respectively $k$ determine how many subgroups are mined by the beam search (first phase) respectively how many subgroups are selected in the end (third phase). The mincov and maxdepth parameters impose a minimum coverage on each subgroup and a maximum depth on the entire search space. Finally, $P$ is a set of parameters that depends on the specific subgroup selection strategy that is used; the strategy could be considered part of $P$. Whenever a fixed-size selection scheme is used, beam width is the only parameter, i.e. $P=\{w\}$. For the parameters of the variable-size schemes, please refer to the previous section. 


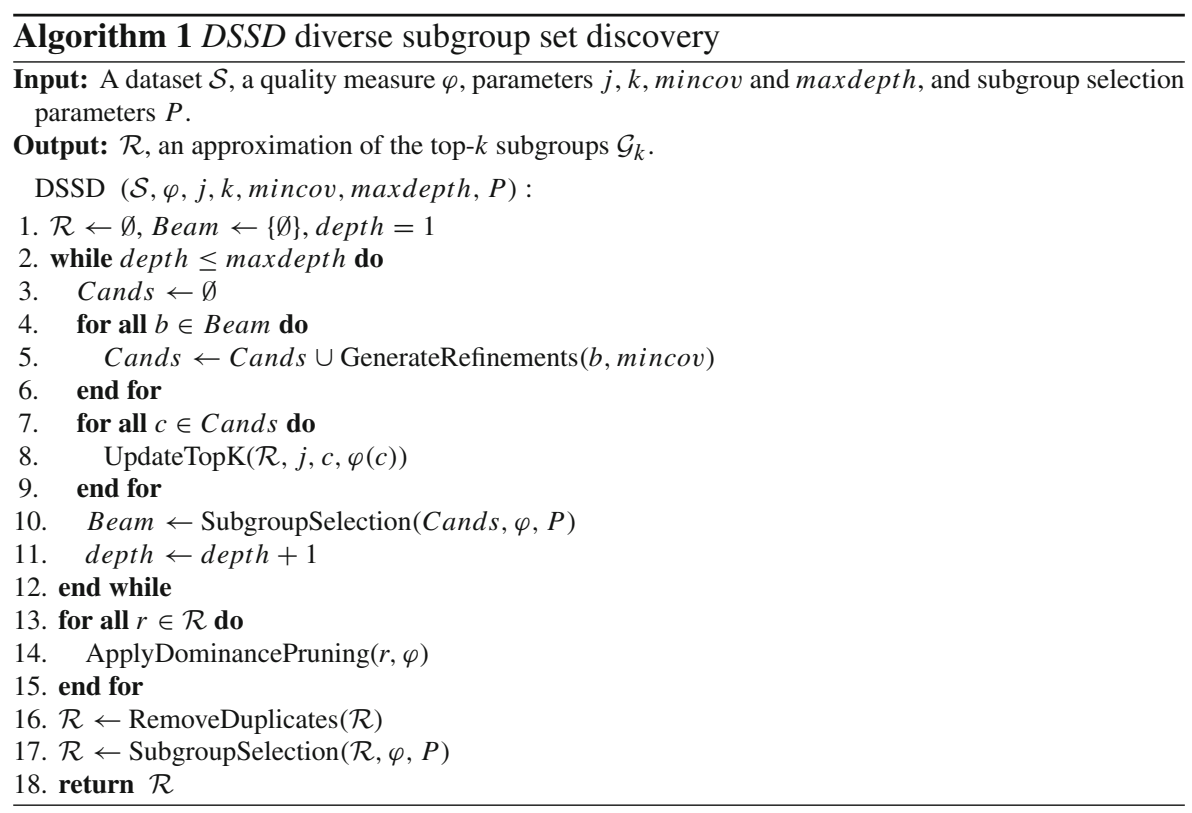

\subsection{Refining subgroups}

Any search method that traverses the search space top-down, refines subgroups by adding conditions to the description one by one. We apply an refinement operator (Algorithm 1, line 5) that, given a subgroup $G$, generates all valid subgroup descriptions that extend $G$ 's description with one condition. We distinguish three types of description attributes, each with its own specifics.

Binary attribute $\{=\}$

The only allowed condition type is 'equals', and consequently only a single condition on any binary attribute can be part of a subgroup description.

Nominal attribute $\{=, \neq\}$

Both 'equals' and 'not equals' are allowed. For any nominal attribute, either a single $=$ or multiple $\neq$ conditions are allowed in a description. (Obviously, $\neq$ conditions cannot specify the same attribute-value.)

Numeric attribute $\{<,>\}$

Both 'less than' and 'greater than' are allowed. Due to the large cardinality of numeric data, generating all possible conditions is infeasible. Thus, to prevent the search space from exploding, the values of a numeric attribute that occur within a subgroup are binned into six equal-sized bins and $\{<,>\}$-conditions are generated for the five cut points obtained this way. This 'on-the-fly' discretisation, performed upon refinement of a subgroup, results in a much more fine-grained binning than a priori discretisation. Multiple conditions on the same attribute are allowed, even though this may lead to redundant conditions in a description (e.g. $D_{x}<10 \wedge D_{x}<5$ ). Dominance-based pruning eradicates this problem though (see next subsection). 
Note that multiple conditions on the same attribute are allowed for nominal and numeric data; slowly peeling off tuples can be helpful to guide the search towards high-quality subgroups.

\subsection{Improving individual subgroups using dominance}

Despite all efforts to prevent and eliminate redundancy in the result set $R$, some of the found subgroups may be overly specific. This may be caused by a large search depth, but also by heuristic choices in e.g. the refinement operator. For example, the subgroup corresponding to $A=$ true $\wedge B=$ true might have the highest possible quality, but never be found since neither $A=$ true nor $B=$ true has high quality. However, $C=$ false $\wedge A=$ true $\wedge B=$ true could be found. Now, pruning the first condition would give the best possible subgroup.

We propose to improve individual subgroups by pruning the subgroup descriptions as a post-processing step, based on the concept of dominance. A subgroup $G_{i}$ dominates a subgroup $G_{j}$ iff

1. the conditions of the description of $G_{i}$ are a strict subset of those of $G_{j}$, and

2. the quality of $G_{i}$ is higher than or equal to that of $G_{j}$, i.e. $\varphi\left(G_{i}\right) \geq \varphi\left(G_{j}\right)$.

Observe that although dominance is clearly inspired by relevancy (Garriga et al 2008), it is not the same. Our definition of dominance is more generic, making it suitable for all target types, e.g. for EMM.

The heuristic method we propose for dominance-based pruning is to consider each of the conditions in a subgroup description one by one, in the order in which they were added. If removing a condition does not decrease the subgroup's quality, then permanently remove it, otherwise keep it.

\section{Experiments}

In this section we report on over 300 experiments that were performed to evaluate DSSD, and to compare it to existing approaches. For this, the following ten different search strategies were used. For each strategy, its full name and an abbreviation (between brackets) are given.

\section{$D S S D$}

DSSD-Description (Desc)—With fixed-size description-based selection.

DSSD-VarDescription (VarDesc) —With variable-size description-based selection. Each attribute may occur $c=2$ times, $l=$ current depth.

DSSD-Cover (Cover) -With fixed-size cover-based selection. Weight parameter $\alpha=0.9$ to give a good balance between quality and diversity.

DSSD-VarCover (VarCover) — With variable-size cover-based selection. The minimum score fraction is set to $f=0.5$.

DSSD-Compression (Comp) -With fixed-size compression-based selection. DSSD-VarCompression (VarComp) —With variable-size compression-based selection. 


\section{Beam search}

SDBeamSearch (Beam)—Standard SD beam search, mines $k$ subgroups.

SDBeamSearch + Post-Processing $($ Beam $+P P)$-As Beam, except that $j$ subgroups are mined, on which post-processing is applied: first dominance pruning, then fixedsize cover-based selection to select $k$ subgroups.

SDBeamSearch with sequential covering (Beam-Seq, Sequential)—The standard beam search is iteratively applied using sequential covering, until $k$ subgroups are found or fewer than minCov tuples remain.

SDBeamSearch with weighted covering (Beam-Weighted, Weighted)-The standard beam search is iteratively applied using multiplicative weighted covering, until $k$ subgroups are found. $\gamma=0.9$, since it is closely related to Cover's $\alpha$. Note that our implementation of weighted covering differs slightly from the one originally proposed in Lavrač et al (2004), as that assumes the single binary target attribute setting. Here, we maintain weights for all tuples (irrespective of their target attribute-values). Then, to compute the score of a subgroup, we calculate the average weight over all tuples in a subgroup and multiply the subgroup's quality with this average weight.

Exhaustive search

Depth-first search $(D F S)$-A standard depth-first search to directly mine the top$k$ subgroups. WRAcc is used in combination with its tight optimistic estimate (Grosskreutz et al 2008). Multiple conditions on a single attribute are allowed, but all attributes are considered in a fixed order to limit the size of the search space. This also means that beam search can potentially reach better solutions.

Depth-first search + Post-Processing $(D F S+P P)$-As $D F S$, except that the same post-processing as with $B e a m+P P$ is applied.

Beam, Beam-Seq, Beam-Weighted and DFS directly mine the $k$ highest quality subgroups. All other strategies first mine $j=10,000$ subgroups, from which $k=100$ are selected for the final subgroup set. A maximum depth maxdepth $=5$ and minimum coverage mincov $=10$ are used. For all fixed-size beams, beam width $w=100$ is used. Preliminary experiments showed that changing these parameters has the same effect on all search strategies, keeping their differences intact. Since our aim is to compare the different strategies, we keep these fixed.

Implementation All proposed and used methods in this paper have been implemented in $\mathrm{C}++$, and both binaries and code are publicly available on the web. ${ }^{2}$ All experiments were conducted on a quad-core Xeon $3.0 \mathrm{GHz}$ system with $8 \mathrm{~Gb}$ of memory running Windows Server 2003. Each run was allowed to use (at most) 24 h computation time on a single core, using (at most) 2 Gb of memory; experiments that did not adhere to these restrictions were terminated. See Table 7 in Appendix A for a complete list of experiments that did and did not meet these resource limitations. Finally, fixed-size cover-based subgroup selection has also been implemented in Cortana $\mathrm{SD},{ }^{3}$ an open source implementation in Java that can be used for various types of SD and EMM.

\footnotetext{
2 http://www.patternsthatmatter.org/dssd/.

3 http://datamining.liacs.nl/cortana.html.
} 
Table 2 Datasets

\begin{tabular}{|c|c|c|c|c|c|c|}
\hline Dataset & $|\mathcal{S}|$ & $\left|D_{b i n}\right|$ & $\left|D_{\text {nom }}\right|$ & $\left|D_{\text {num }}\right|$ & $|M|$ & \\
\hline \multicolumn{7}{|l|}{ Single binary } \\
\hline Adult-SD & 48,842 & 99 & - & 6 & 1 & \\
\hline Credit-G & 1,000 & 2 & 11 & 7 & 1 & \\
\hline Mushroom & 8,124 & 4 & 18 & - & 1 & \\
\hline COIL2000 & 5,822 & 5 & 80 & - & 1 & \\
\hline Single nominal & & & & & & $\left|\operatorname{Dom}\left(M_{1}\right)\right|$ \\
\hline Car & 1,728 & - & 6 & - & 1 & 4 \\
\hline Nursery & 12,960 & - & 8 & - & 1 & 5 \\
\hline \multicolumn{7}{|l|}{ Single numeric } \\
\hline Abalone & 4,177 & - & 1 & 7 & 1 & \\
\hline Crime & 1,994 & - & 1 & 101 & 1 & \\
\hline Elections-SD & 1,846 & 4 & 67 & 1 & 1 & \\
\hline Red-wine & 1,599 & - & - & 11 & 1 & \\
\hline White-wine & 4,898 & - & - & 11 & 1 & \\
\hline Multiple binary & & & & & & $W K G$ minsup \\
\hline Adult-EMM & 48,842 & - & - & 6 & 99 & $10 \%$ \\
\hline CAL500 & 502 & - & - & 68 & 174 & - \\
\hline Emotions & 593 & - & - & 72 & 6 & $1 \%$ \\
\hline Mammals & 2,221 & - & - & 67 & 124 & - \\
\hline Yeast & 2417 & - & - & 103 & 14 & $1 \%$ \\
\hline \multicolumn{7}{|l|}{ Multiple nominal } \\
\hline Elections-EMM & 1,846 & 4 & 5 & 1 & 62 & \\
\hline
\end{tabular}

For each dataset the number of tuples, the number of binary, nominal, and numeric description attributes, and the number of model attributes are given. Further, for the single nominal datasets the number of distinct target values is given, and for the multiple binary case the minsup used for $W K G$ is given

\subsection{Datasets}

To evaluate the proposed methods, we perform experiments on the datasets listed in Table 2. In this table, the datasets are grouped by model/target type. The CAL500, Emotions and Yeast datasets were taken from the 'Mulan' repository ${ }^{4}$ (Tsoumakas et al 2010). Further, we use the Mammals dataset (Heikinheimo et al 2007), which consists of presence information of European mammals (Mitchell-Jones et al 1999) and climate information.

The two Elections datasets were constructed from data collected by the 'election engine' at www.vaalikone.fi before the 2011 parliamentary elections in Finland. The data was published by Helsingin Sanomat, ${ }^{5}$ a Finnish newspaper, and it consists of information about the roughly 2000 candidates that participated in the elections. In

\footnotetext{
4 http://mulan.sourceforge.net/datasets.html.

5 http://blogit.hs.fi/hsnext/hsn-vaalikone-on-nyt-avointa-tietoa.
} 
the EMM-variant of the dataset, candidate properties such as party, age, and education are used as description attributes, while the answers and weights assigned by the candidates to 30 questions are used as model data. For each question, the candidates could choose one from 3 up to 8 answers. In the SD-variant of the dataset, all attributes just mentioned are used as description data, and the number of votes each candidate received in the elections is used as target. In that case, the goal of SD can be interpreted to be finding candidate properties and answers that result in relatively many votes.

The rest of the datasets are taken from the UCI repository. ${ }^{6}$ Two variants of the UCI Adult dataset are used: Adult-SD is the commonly used variant, with the binary class label as single target, in Adult-EMM all numeric attributes are considered as description attributes, and all binary attributes as model attributes (except for class, which is not used). For the Crime dataset, the unprocessed 'unnormalized' version was taken and pre-processed as follows. First, all non-predictive attributes and potential goals except for violentPerPop were removed. Next, all tuples that have a missing value for violentPerPop were removed. Finally, all attributes with missing values were removed.

\subsection{A characteristic multiple model attribute experiment in detail}

To study the effects of the proposed search strategies and dominance-based pruning in detail, we focus on a single dataset. As we previously presented a detailed SD example in van Leeuwen and Knobbe (2011), we here present an example with multiple model attributes. For ease of presentation, we choose the relatively small Emotions dataset with WKL as quality measure. From Fig. 1 we have already seen that redundancy in the subgroup covers is a problem when a standard beam search is used, and we will now investigate how the newly proposed search strategies improve diversity.

Figure 2 shows which subgroups are selected for refinement on each level in the beam search performed by DSSD. Clearly, all selection strategies select subgroups from a much wider range than the standard top-100, which is likely to result in more diverse beams. Looking at the upper three plots, we observe that a higher degree of redundancy elimination results in more (high-quality but similar) candidates being skipped; this fully meets our expectations.

Our hypothesis is that the diverse beam selection methods result in more diverse (and therefore less redundant) subgroup sets. To assess this, consider the subgroup covers depicted in Fig. 3. Compared to the Beam results shown in Fig. 1, it is clear that mining more subgroups and adding a post-processing phase helps to improve diversity (see Beam $+P P$ ). Actually, both a visual inspection and the values for CR and $\mathrm{H}$ reveal that the results obtained this way are quite similar to those obtained with Description. The latter is faster though (117s vs $238 \mathrm{~s})$ and finds a top-1 subgroup with higher quality ( $W K L=0.60$ vs $W K L=0.56$ ).

\footnotetext{
6 http://archive.ics.uci.edu/ml/.
} 

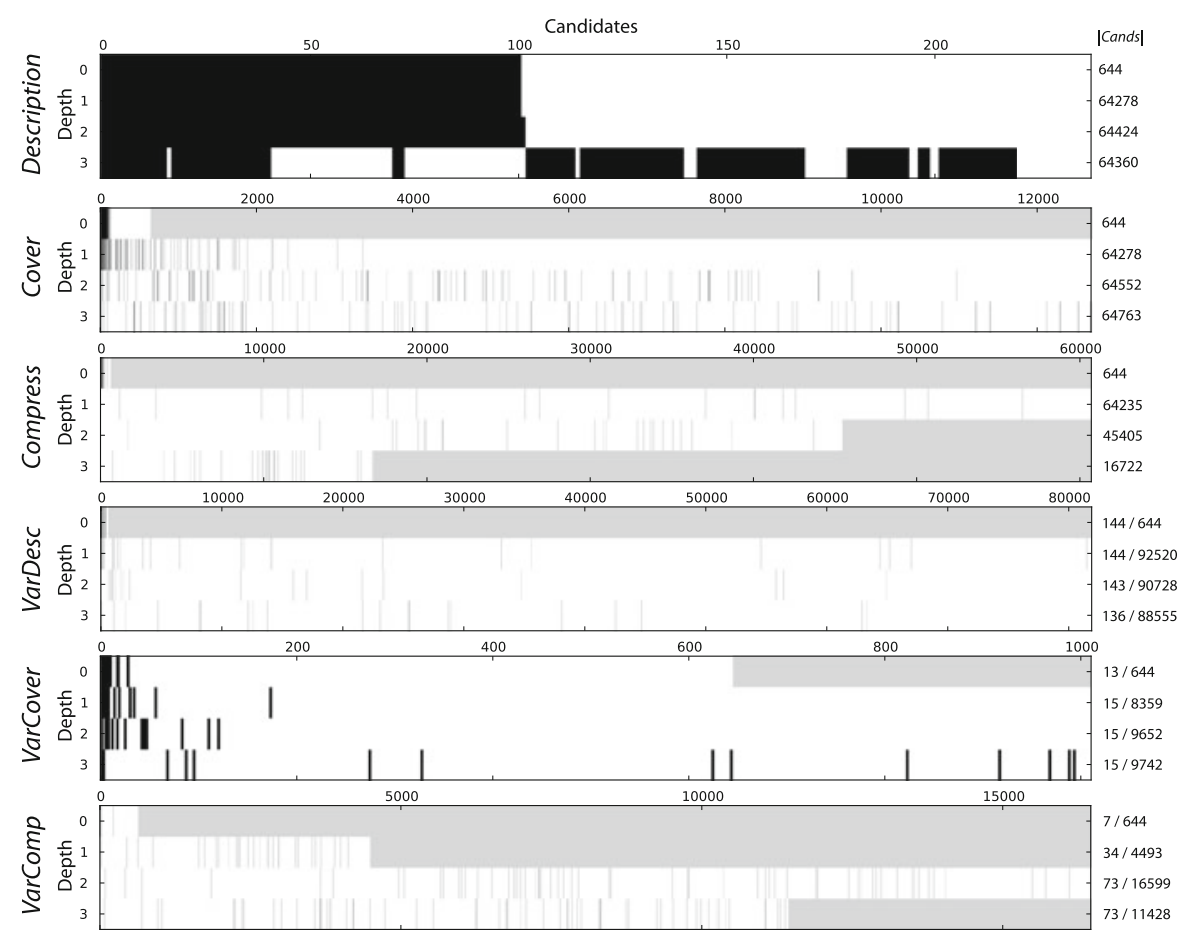

Fig. 2 Different DSSD subgroup selection strategies in action, on dataset Emotions with WKL as quality measure. For each level in the beam search, it is shown which candidate subgroups are selected for inclusion in the beam (black) and which are ignored (white). Candidates are ordered descending on quality. On the right, the total number of candidate subgroups for each level is shown (candidates not shown are not selected). In the upper three plots, $w=100$ subgroups are selected on each level, while the number of selected subgroups is shown on the right for the lower three plots

Both Cover and Compress further improve subgroup cover diversity, but do so in different ways. Cover discovers subgroup sets consisting of quite large subgroups (containing 202.1 tuples on average), while Compress finds much smaller subgroups (78.5 tuples on average). The cover-based approach is much more diverse in terms of $\mathrm{CR}$, but the joint entropy of the results of the compression-based method is also quite high.

Then there are the sequential and weighted covering approaches. Sequential covering finds only 19 subgroups, after which the resulting dataset consists of too few tuples to continue $(|\mathcal{S}|<=\operatorname{minCov})$. The Weighted approach finds results that seem quite similar - and are possible even more diverse - than those obtained with Cover. The subgroups are of similar size (211.5 tuples on average), the best subgroup found is of almost the same quality (but slightly lower), and both $\mathrm{CR}$ and $\mathrm{H}$ indicate slightly more diverse subgroup covers. There is one important difference however, as Cover needed only 4 min to run, while the weighted covering approach needed $112 \mathrm{~min}$. While achieving similar results, the cover-based strategy provides a significant $28 \times$ speed-up with respect to weighted covering! 


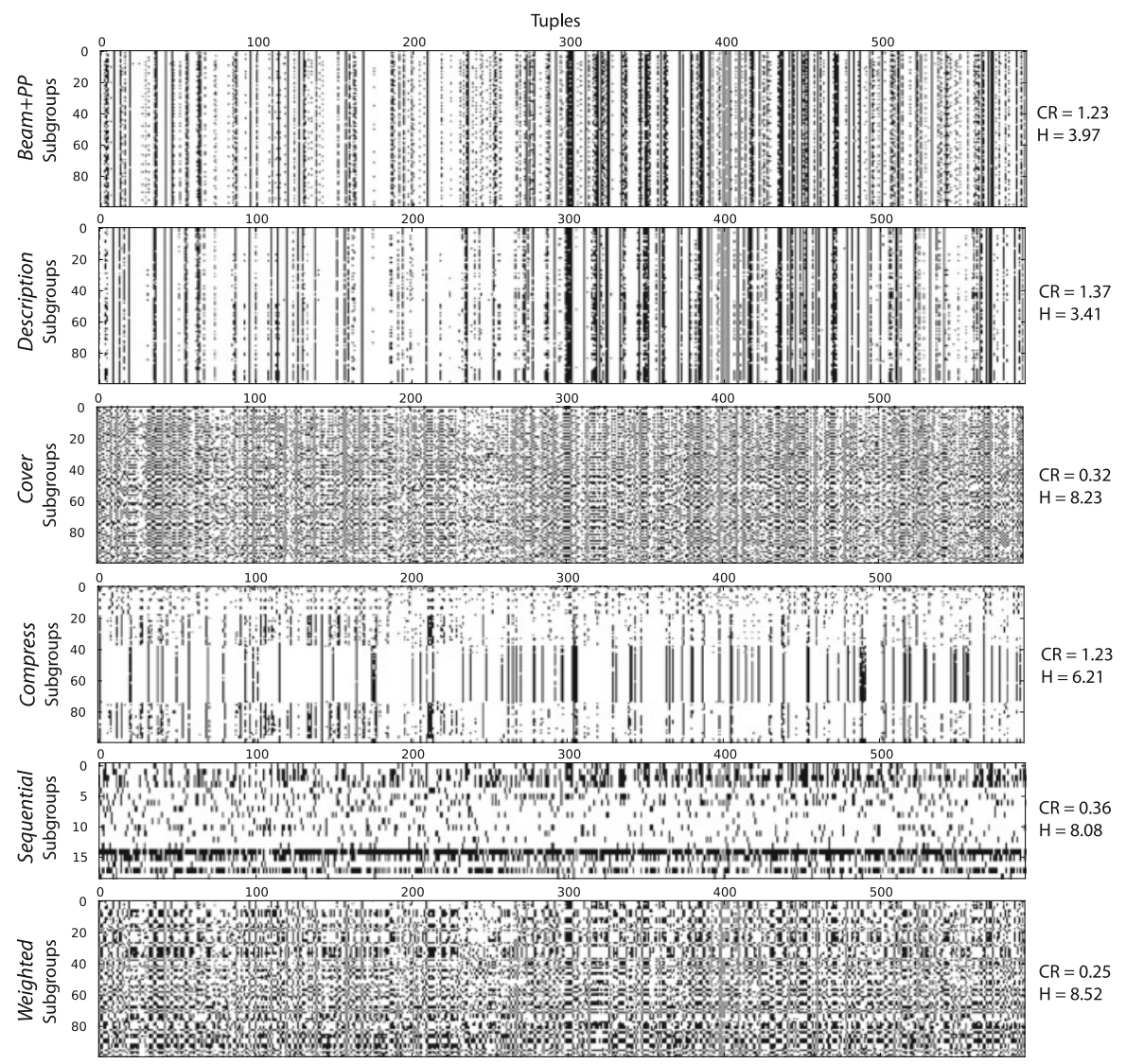

Fig. 3 Subgroup covers obtained with six search strategies. Shown are the covers (in black) of the subgroups obtained on Emotions with WKL. CR and joint entropies $(\mathrm{H})$ calculated on the subgroup sets are shown on the right

In Sect. 4 we stated that it is our goal to find non-redundant sets of high-quality subgroups. It is therefore important that the maximum quality of a subgroup set, the highest quality obtained by any subgroup, is as high as possible. To assess this, consider the qualities of the obtained subgroup sets depicted in Fig. 4. All alternative approaches clearly give more diverse results than Beam, as we already saw from the subgroup covers; the lower average qualities and larger standard deviations are natural consequences of the diversity enforced by subgroup set selection. Weighted, Description and Cover attain higher maximum qualities than the rest, confirming that diversity may contribute to find higher quality subgroups. Sequential covering and the compression-based approaches do not seem to be good alternatives when high quality is a primary requirement.

Finally, let us consider an example of the effect of dominance-pruning. After the first phase of DSSD, the beam search, the descriptions of the 10,000 discovered subgroups consist of 43,258 conditions in total. After applying the pruning phase, this has 


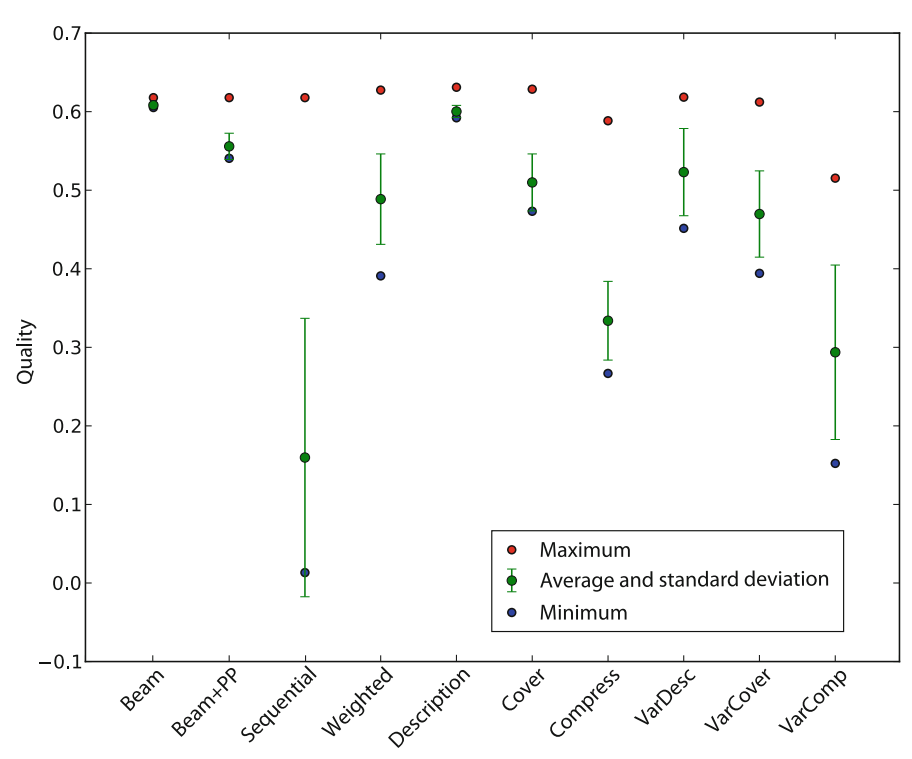

Fig. 4 Qualities of subgroup sets obtained with different search strategies

been reduced to 34,706 conditions, meaning that 8,552 conditions could be removed! Meanwhile, average quality has increased from 0.509 to 0.522 . This clearly demonstrates the usefulness of dominance-based pruning of individual subgroups; subgroup descriptions become shorter and thus simpler, while quality increases.

\subsection{Quantitative results}

We now present results obtained on a large number of experiments performed on all datasets, to show when it may be beneficial to use the DSSD algorithm, and when it is better to use existing methods. Primary objectives are to discover subgroup sets that are (1) high quality and (2) diverse in (3) as little computation time as possible. Results are aggregated per target type, with the multiple binary and nominal model attribute types combined.

Regarding the first two objectives, a search strategy is better than others if it more often achieves (1) a higher maximum quality, (2) a lower CR, and (3) a higher joint entropy. We quantify this using average rank results. For each combination of dataset, quality measure and search strategy, an experiment was conducted and ranked with respect to (1) maximum quality ( $\varphi^{\max }$, descending), (2) CR (ascending), and (3) joint entropy (H, descending), stratified by search strategy. Tied ranks are assigned the average of the ranks for the range they cover. Finally, all ranks for a specific search strategy are averaged.

Results obtained for the single binary setting are shown in Table 3. Of all search strategies, Beam, DFS and DFS $+P P$ result in most redundancy, and the depth-first search strategies also reach less high quality (due to the fixed order in which attributes 
Table 3 The single binary case, aggregated over Adult-SD, Credit-G, Mushroom, and COIL2000 with WRAcc and WKL

\begin{tabular}{|c|c|c|c|c|c|c|c|c|c|}
\hline \multirow[t]{2}{*}{ Search strategy } & \multicolumn{2}{|l|}{ Exp avg } & \multicolumn{4}{|c|}{ Subgroup set avg } & \multicolumn{3}{|c|}{ Rank avg } \\
\hline & $\mathrm{t}(\min )$ & $|\mathcal{R}|$ & Descr. & Size & $\mathrm{CR}$ & $\mathrm{H}$ & $\varphi^{\max }$ & $\mathrm{CR}$ & $\mathrm{H}$ \\
\hline DSSD-Desc & 21.1 & 94 & 4.6 & 4328 & 1.18 & 2.36 & 4.3 & 5.7 & 5.0 \\
\hline DSSD-VarDesc & 18.8 & 25 & 4.3 & 1161 & 1.07 & 3.47 & 5.4 & 5.2 & 4.8 \\
\hline DSSD-Cover & 59.8 & 100 & 4.3 & 5642 & 0.63 & 5.01 & 4.3 & 2.7 & 2.9 \\
\hline DSSD-VarCover & 1.8 & 26 & 3.3 & 758 & 11.04 & 3.37 & 4.8 & 7.1 & 4.6 \\
\hline Beam & 11.4 & 100 & 4.8 & 4280 & 1.33 & 1.17 & 4.7 & 7.6 & 8.3 \\
\hline Beam $+P P$ & 12.7 & 100 & 4.0 & 4986 & 1.03 & 2.43 & 4.7 & 5.0 & 5.8 \\
\hline Beam-Seq & 72.2 & 27 & 3.7 & 3224 & 0.35 & 6.28 & 4.7 & 2.0 & 3.4 \\
\hline Beam-Weighted & 427.9 & 100 & 4.8 & 6290 & 0.35 & 7.58 & 4.3 & 1.9 & 1.6 \\
\hline$D F S$ & 551.0 & 100 & 4.9 & 2067 & 1.16 & 1.55 & 7.4 & 8.0 & 8.8 \\
\hline$D F S+P P$ & 577.8 & 100 & 3.3 & 2099 & 1.03 & 2.44 & 7.4 & 7.0 & 8.0 \\
\hline
\end{tabular}

The following averages over all experiments are shown: the time (in minutes) and the average number of discovered subgroups, the number of conditions per subgroup description (descr.), subgroup sizes, CR and joint entropy H. Description and subgroup sizes are first averaged per subgroup set resulting from an experiment, and then averaged over all experiments. On the right, average ranks are given as obtained by ranking experiments stratified by search strategy, for maximum quality, CR and joint entropy

are refined). Beam-Seq, VarDesc and VarCover result in a similar number of subgroups; Beam-Seq gives larger subgroups that have more diverse covers, but the variable-size approaches are much faster.

Cover and Weighted achieve high-quality and quite diverse subgroup sets (in terms of $\mathrm{CR}$ and $\mathrm{H}$ ), but the former is roughly seven times faster on average. The fixedsize description-based approach seems a reasonable alternative if one wants results quickly, as it attains high quality results in little time.

Table 4 shows that runtimes, subgroup sizes and maximum qualities are quite comparable among the search strategies in the single nominal setting. Desc seems to perform very well in terms of cover diversity, while CR and joint entropy for BeamWeighted are rather inconclusive.

The results of the single numeric case, presented in Table 5, are more interesting, as the differences are larger. Weighted covering seems to do a very good job: the maximum qualities rank quite high and both the average ranks for $\mathrm{CR}$ and $\mathrm{H}$ are outstanding. However, the runtime is problematic when compared to Cover, exhibiting a staggering difference of a factor 23. CR and joint entropy obtained with cover-based selection indicate a bit less diversity, but the difference does not seem too large and Cover does rank higher with respect to maximum quality. Also, it is important to observe that Cover does better than Beam $+P P$, except that it is slower.

We previously observed that sequential covering gives few yet very diverse subgroups of reasonable quality. However, it isn't particularly fast and we know from its definition that it discourages overlapping subgroups. This makes the discovery of many possibly interesting subgroups highly unlikely and we therefore discard sequential covering as a serious competitor. The VarDesc and VarCover approaches also return 
Table 4 The single nominal case, aggregated over Car and Nursery with $\chi^{2}, M W R A c c$ and WKL

\begin{tabular}{|c|c|c|c|c|c|c|c|c|c|}
\hline \multirow[t]{2}{*}{ Search strategy } & \multicolumn{2}{|c|}{ Exp avg } & \multicolumn{4}{|c|}{ Subgroup set avg } & \multicolumn{3}{|c|}{ Rank avg } \\
\hline & $\mathrm{t}(\min )$ & $|\mathcal{R}|$ & Descr. & Size & $\mathrm{CR}$ & $\mathrm{H}$ & $\varphi^{\max }$ & CR & $\mathrm{H}$ \\
\hline DSSD-Desc & 0.3 & 60 & 2.4 & 2649 & 0.31 & 8.27 & 5.4 & 3.3 & 2.3 \\
\hline DSSD-VarDesc & 0.0 & 14 & 2.0 & 2716 & 0.35 & 6.06 & 5.4 & 5.2 & 5.3 \\
\hline DSSD-Cover & 3.7 & 100 & 2.4 & 2640 & 0.48 & 5.96 & 5.4 & 5.2 & 5.0 \\
\hline DSSD-VarCover & 0.0 & 13 & 2.5 & 1264 & 1.87 & 3.15 & 6.3 & 9.7 & 8.2 \\
\hline Beam & 0.0 & 100 & 3.4 & 2116 & 1.00 & 4.61 & 5.4 & 7.7 & 6.2 \\
\hline Beam $+P P$ & 1.5 & 100 & 2.2 & 2655 & 0.59 & 5.82 & 5.4 & 6.4 & 5.2 \\
\hline Beam-Seq & 0.0 & 16 & 1.7 & 1738 & 0.25 & 9.30 & 5.4 & 3.6 & 1.8 \\
\hline Beam-Weighted & 0.5 & 100 & 1.3 & 3547 & 0.10 & 1.84 & 5.4 & 1.2 & 9.5 \\
\hline$D F S$ & 0.5 & 100 & 3.5 & 2003 & 0.87 & 7.12 & 5.4 & 7.8 & 4.5 \\
\hline$D F S+P P$ & 3.5 & 100 & 2.7 & 2396 & 0.39 & 4.83 & 5.4 & 5.1 & 6.5 \\
\hline
\end{tabular}

See Table 3 for a description of the columns

Table 5 The single numeric case, aggregated over Abalone, Crime, Elections-SD, Red-wine, and Whitewine with NWRAcc and MT

\begin{tabular}{|c|c|c|c|c|c|c|c|c|c|}
\hline \multirow[t]{2}{*}{ Search strategy } & \multicolumn{2}{|l|}{ Exp avg } & \multicolumn{4}{|c|}{ Subgroup set avg } & \multicolumn{3}{|c|}{ Rank avg } \\
\hline & $\mathrm{t}(\min )$ & $|\mathcal{R}|$ & Descr. & Size & $\mathrm{CR}$ & $\mathrm{H}$ & $\varphi^{\max }$ & $\mathrm{CR}$ & $\mathrm{H}$ \\
\hline DSSD-Desc & 9.1 & 100 & 3.8 & 963 & 1.29 & 2.31 & 4.5 & 6.6 & 6.1 \\
\hline DSSD-VarDesc & 20.9 & 26 & 3.2 & 891 & 1.10 & 4.28 & 5.4 & 5.4 & 5.3 \\
\hline DSSD-Cover & 18.2 & 100 & 3.2 & 1494 & 0.74 & 7.00 & 3.7 & 3.1 & 2.7 \\
\hline DSSD-VarCover & 0.1 & 16 & 2.8 & 554 & 2.67 & 3.04 & 6.4 & 8.0 & 6.0 \\
\hline Beam & 8.2 & 100 & 4.8 & 987 & 1.34 & 1.16 & 5.6 & 8.1 & 8.9 \\
\hline Beam $+P P$ & 5.4 & 100 & 3.2 & 1210 & 1.04 & 4.65 & 4.7 & 4.6 & 4.1 \\
\hline Beam-Sequential & 75.7 & 23 & 4.4 & 1072 & 0.26 & 7.90 & 5.6 & 1.0 & 3.3 \\
\hline Beam-Weighted & 412.3 & 100 & 4.3 & 1398 & 0.47 & 9.31 & 4.9 & 2.1 & 1.5 \\
\hline$D F S$ & 40.7 & 100 & 4.6 & 1330 & 1.23 & 1.27 & 6.4 & 8.4 & 9.2 \\
\hline$D F S+P P$ & 42.7 & 100 & 2.9 & 1529 & 0.94 & 3.66 & 4.9 & 5.8 & 6.7 \\
\hline
\end{tabular}

See Table 3 for a description of the columns

small subgroup sets, but these are faster than Sequential and do not discourage subgroups. The downside is that the results do not stand out with respect to either quality or cover diversity.

We now switch from the classical SD to the EMM setting, where we have multiple model attributes. Table 6 summarises the results obtained using the WKL quality measure. The highest qualities are obtained with the Desc and Weighted approaches. The latter achieves far more diverse results, but also needs much more time for this. The Cover approach seems a good compromise, as it is 20 times faster than Weighted, and finds subgroups of reasonably high quality and diversity. Again, it outperforms $B e a m+P P$ on all objectives except for speed. The compression-based strategies take 
Table 6 The multiple binary or nominal case with $W K L$ as quality measure, aggregated over Adult-EMM, CAL500, Elections-EMM, Emotions, Mammals, and Yeast

\begin{tabular}{|c|c|c|c|c|c|c|c|c|c|}
\hline \multirow[t]{2}{*}{ Search strategy } & \multicolumn{2}{|l|}{ Exp avg } & \multicolumn{4}{|c|}{ Subgroup set avg } & \multicolumn{3}{|c|}{ Rank avg } \\
\hline & $\mathrm{t}(\min )$ & $|\mathcal{R}|$ & Descr. & Size & $\mathrm{CR}$ & $\mathrm{H}$ & $\varphi^{\max }$ & $\mathrm{CR}$ & $\mathrm{H}$ \\
\hline DSSD-Desc & 3.7 & 85 & 3.6 & 2022 & 1.43 & 2.64 & 3.8 & 7.9 & 7.9 \\
\hline DSSD-VarDesc & 19.0 & 43 & 3.3 & 1365 & 1.06 & 3.87 & 4.8 & 5.8 & 6.2 \\
\hline DSSD-Cover & 9.7 & 86 & 3.2 & 2975 & 0.68 & 6.53 & 5.6 & 3.6 & 3.3 \\
\hline DSSD-VarCover & 0.5 & 33 & 2.3 & 688 & 3.78 & 5.62 & 6.1 & 8.0 & 4.2 \\
\hline DSSD-Compress & 134.8 & 100 & 2.3 & 299 & 1.34 & 4.32 & 7.4 & 6.7 & 5.7 \\
\hline DSSD-VarComp & 124.8 & 21 & 2.4 & 723 & 0.76 & 3.69 & 8.3 & 4.2 & 6.2 \\
\hline Beam & 1.2 & 100 & 4.7 & 2916 & 1.57 & 1.36 & 4.9 & 8.8 & 10.0 \\
\hline Beam $+P P$ & 3.5 & 85 & 3.3 & 2903 & 1.20 & 3.58 & 5.8 & 6.5 & 6.4 \\
\hline Beam-Seq & 47.2 & 32 & 4.1 & 1979 & 0.29 & 8.80 & 4.9 & 2.0 & 1.8 \\
\hline Beam-Weighted & 192.5 & 100 & 3.5 & 2931 & 0.27 & 6.64 & 3.5 & 1.5 & 3.3 \\
\hline
\end{tabular}

See Table 3 for a description of the columns

a long time but do not seem to result in either high quality or high diversity. It results in smaller subgroups with shorter subgroup descriptions though, which may be useful for specific applications.

Finally, we tested which experiments could be performed with $W K G$ quality. This measure is the most demanding of all, and turned out infeasible on CAL500 and Mammals within the imposed resource limits. Table 7 in the Appendix shows which experiments did finish within these limits. Since only few experiments finished, we do not present the aggregated results. For the three datasets for which it was possible to experiment with $W K G$, the Cover strategy always finished, and did so in $119.7 \mathrm{~min}$ on average. Its main competitor, weighted covering, was not able to run on two out of three of these datasets. For Adult-EMM, on which it did finish, it needed 1339 min-just within the time limit of a day.

To conclude this subsection, several observations can be made. First and foremost, it is important to observe that the fixed-size cover-based strategy always performs better than Beam $+P P$ in terms of diversity, and often achieves a higher maximum quality. It is somewhat slower, but runtimes are manageable. This suggests that incorporating subgroup selection within beam search yields clearly better results than applying it as post-processing step. Compared to weighted covering, it achieves slightly less diverse results in far less time.

Employing the fixed-size description-based selection scheme comes at little computational cost, but does give higher-quality and more diverse results than without using any subgroup selection techniques. The compression-based method does not always work well and is slow, but should be employed for datasets where many underlying distributions are present in the model data, such as it is the case for e.g. Mammals. 
Although using a variable beam width still seems an interesting and novel idea, the heuristics did not yet convince in these experiments. The resulting strategies can very quickly find small sets of subgroups though, and this may be useful for specific applications.

\section{Related Work}

The development of the SD field has had a comparatively long history, dating back to early work by e.g. Webb (1995), Klösgen (1996, 2002) and Wrobel (1997). This research has produced tools for supervised pattern discovery such as Explora (Klösgen 1996) and Opus (Webb 1995). The various publications in the SD field have always had a very applied focus, with SD tools providing informative and interpretable patterns that were of benefit to end-users in various domains, including medicine (Lavrač et al 2004), genomics (Pieters et al 2010), voting behaviour (Grosskreutz et al 2010), etc.

More recently, more theoretical work has emerged (Garriga et al 2008; Grosskreutz et al 2008; Grosskreutz and Paurat 2011; Lemmerich et al 2010), in part inspired by more theoretical work in the unsupervised counterpart of SD, frequent pattern mining. Much of this work focuses on the exhaustive, yet efficient traversal of the subgroup hypothesis space, while pruning parts of the search space that can be proven not to yield candidate subgroups of high quality. Note that much of the work in this area assumes that the available attributes are discrete in nature, and that exhaustive enumeration is in theory feasible. The essential difference with the work proposed here, is that we consider datasets that are too complex to consider in an exhaustive manner. As a result, we will have to resort to a heuristic approach that only considers the most promising parts of the search space.

As for reducing the redundancy in the output, Garriga et al (2008) has proposed closed sets for labeled data, but similarly to closed frequent itemsets, this only eliminates a very specific form of redundancy, as closedness only affects patterns with respect to super-/subsets and the single binary target setting is assumed. Recently, Lemmerich and Puppe (2011) proposed a method for reducing the number of patterns reported by considering their 'expectation' in terms of statistics of contained patterns (that is, ancestors in terms of the search space).

Within the EMM framework, the EMDM algorithm was proposed (van Leeuwen 2010). This approach to EMM is quite different from the classical approaches, both exhaustive and heuristic, for several reasons. First of all, it cannot be used for classical SD (with a single target attribute), since not enough information is available for the exception maximisation step in that situation. Second, EMDM needs a way to do exception maximisation, which depends on the quality measure and may not be possible to devise for each measure. Finally, the approach does not have a maxdepth parameter and results in far more specific subgroups than is common; subgroups tend to be smaller and have more complex descriptions (disjunctions of conjunctions). 
To the best of our knowledge, we are the first to combine pattern selection techniques and beam search to achieve non-redundant GSD. Kocev et al (2007) previously proposed to incorporate similarity constraints in a beam search to improve the induction of predictive clustering trees. The subgroup selection strategies we propose are clearly inspired by pattern set selection methods such as those proposed by Peng et al (2005), Knobbe and Ho (2006a,b), and Bringmann and Zimmermann (2007). The key difference is that we are not only concerned with redundancy within the final result, but rather in achieving a certain level of diversity (Shell et al 1994) during the search process. In other words, pattern selection techniques are employed while searching, rather than as a form of post-processing, as is the case with Knobbe and Ho (2006a,b); Bringmann and Zimmermann (2007).

Regarding our experiments with (single) continuous targets, we are building on work by e.g. Atzmüller and Lemmerich (2009) and Grosskreutz and Rüping (2009) that proposes a range of quality measures for numeric domains (Pieters et al 2010). Our experiments with multiple target attributes are of course based on our previous work on EMM (Leman et al 2008; Duivesteijn et al 2010; van Leeuwen 2010).

A framework very much related to SD is that of contrast set mining (CSM), as introduced by Bay and Pazzani (2001); Bailey and Dong (2007).

The aim of CSM is to employ existing (unsupervised) frequent pattern mining techniques to discovery patterns in two or more groups of data, and then compare results between the multiple pattern sets, in order to highlight the contrast between the sets of data. The pattern quality measure of choice for CSM is known as difference of support (SuppDiff). In Webb et al (2003), and later in Kralj Novak et al (2009), it was observed that the multiple 'groups' in CSM could be interpreted as labels of the data, and that as such, there was an intuitive mapping between the SD and CSM setting. In fact, much of the work in either field has been developing in parallel, without much cross-breeding.

Similar observations were made for another pattern mining setting, Emerging Pattern Mining (Dong et al 1999), which was originally aimed at discovering emerging trends in time-stamped data, by comparing candidate patterns on one version of the data with those on a newer version, which can clearly be mapped to an SD setting. The EP setting is based on the GrowthRate measure, which uses the ratio of supports, rather than the difference.

Other terms which are related to SD and could be considered synonyms are correlated pattern mining (Morishita and Sese 2000; Nijssen et al 2009), change mining (Liu et al 2001), mining closed sets from labeled data (Garriga et al 2008), exception rule mining (Daly and Taniar 2005), impact rules (Webb 2001), bump hunting (Friedman and Fisher 1999), quantitative association rules (Aumann and Lindell 1999).

\section{Conclusions}

Effective and efficient heuristics are crucial for performing discovery tasks in large and complex data. In addition to that, the incredible amount of redundancy in hypothesis 
spaces renders straightforward top- $k$ mining useless. We address these problems by incorporating heuristic pattern set selection methods within a beam search, thereby improving the balance between exploration and exploitation.

We described three degrees of redundancy and introduced two subgroup set selection strategies for each degree. We then incorporated these within a beam search and dubbed this algorithm DSSD. Experiments with both SD and EMM show that the proposed subgroup set discovery algorithm returns high-quality yet diverse results in reasonable runtime. By means of the selection strategies, the data miner is offered a trade-off between redundancy elimination and computation time. On the individual subgroup level, dominance-based pruning simplifies overly specific subgroup descriptions.

Compared to existing approaches, DSSD performs very well and nicely balances subgroup quality, diversity and runtime. Depth-first search only seems an option when the target is not complex (i.e. consists of a single binary or nominal attribute) and the search space can be significantly pruned, e.g. using optimistic estimates. Even then, a post-processing phase is required to eliminate redundant results. Weighted covering seems to be the main competitor of DSSD-Cover, but the latter was shown to be 20 times faster on average.

Particularly when the complexity of the target(s) and quality measure increases, the computational complexity of individual candidate testing also increases. When candidate testing is intensive, it turns out to be beneficial to avoid iterative schemes like weighted covering. Especially in those cases, incorporating pattern selection within the search results in significant speed-ups.

Finally, note that although the proposed subgroup selection methods were primarily proposed to improve beam search, they are generic and can be easily used for other purposes, e.g. for post-processing the results of any search.

In the future, we plan to perform an extensive case study to evaluate how DSSD performs in real-world applications. Also, we believe there are opportunities to improve the trade-off between computation time and diversity by means of better selection strategies, possibly by involving the end-user during the search process to facilitate interactive discovery.

Acknowledgements The authors wish to thank Antti Ukkonen for sharing the Finnish elections data. This research is financially supported by the Netherlands Organisation for Scientific Research (NWO) through the EMM project (number 612.065.822) and a Rubicon grant.

Open Access This article is distributed under the terms of the Creative Commons Attribution License which permits any use, distribution, and reproduction in any medium, provided the original author(s) and the source are credited.

\section{Appendix A: Experiment overview}

See Table 7. 


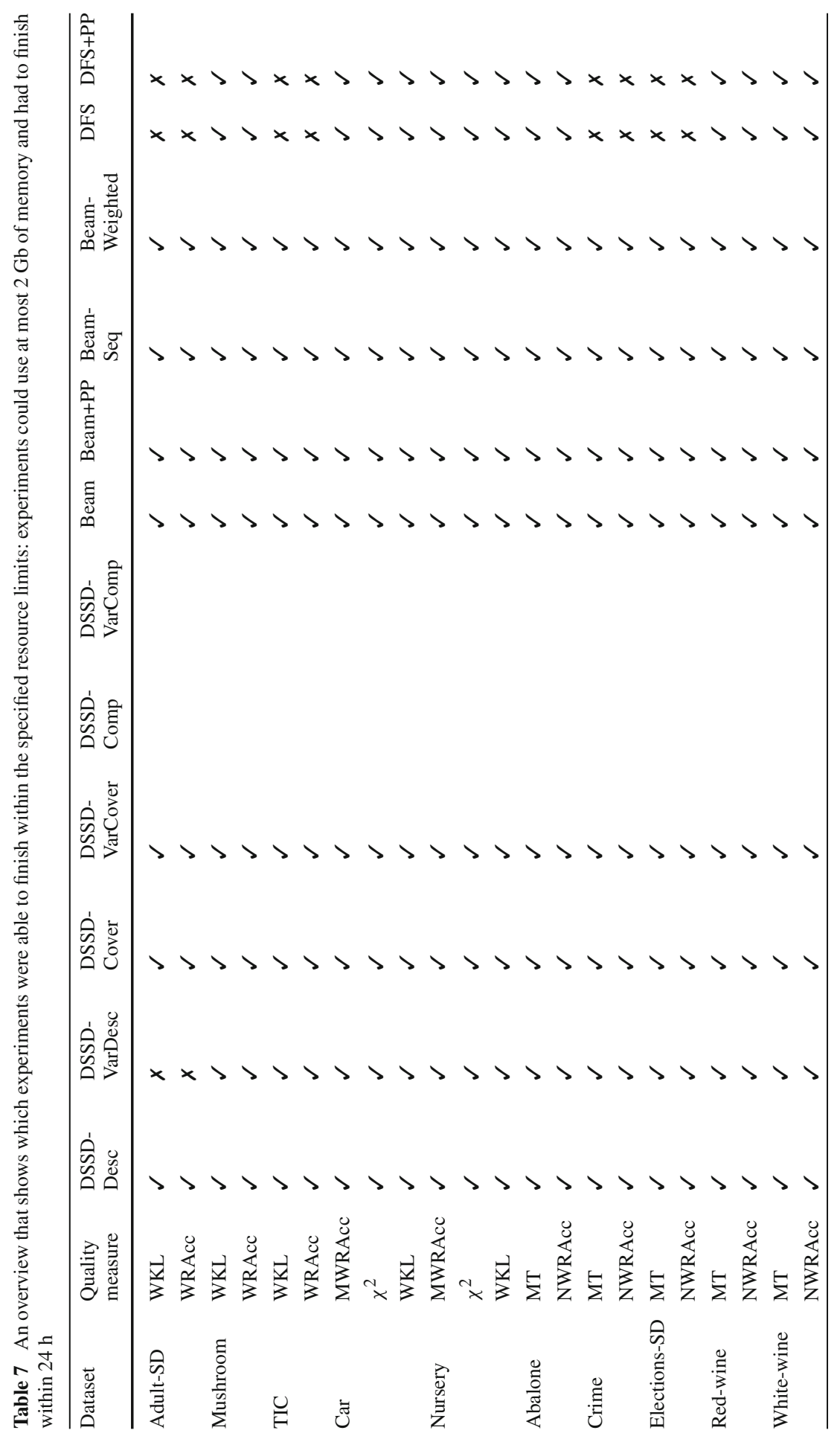




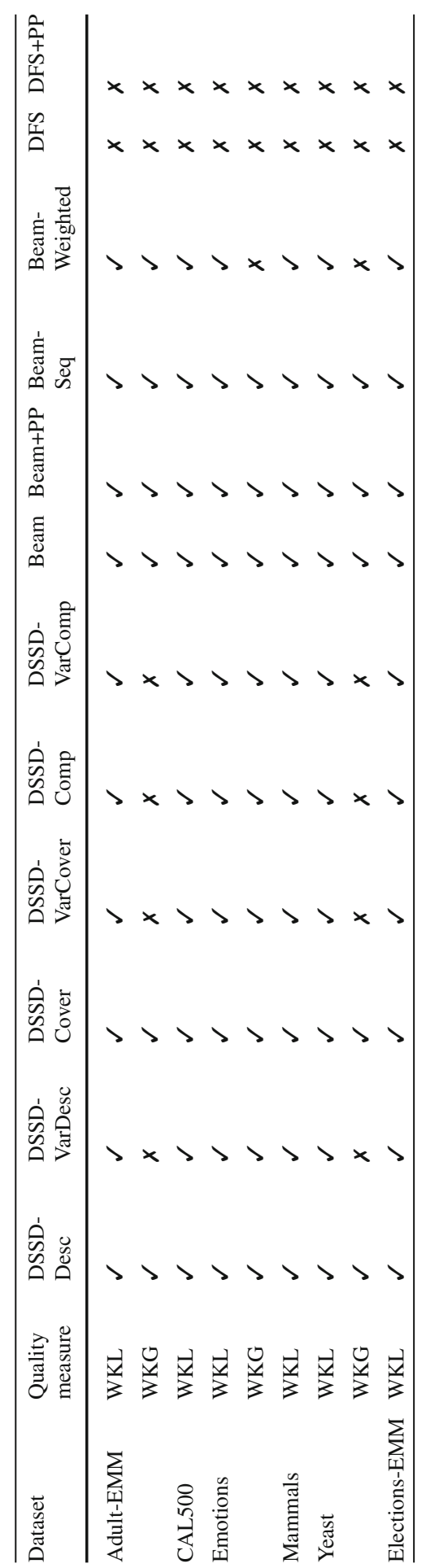




\section{References}

Abudawood T, Flach P (2009) Evaluation measures for multi-class subgroup discovery. In: Proceedings of the ECML/PKDD'09, Bled, pp 35-50

Atzmüller M, Lemmerich F (2009) Fast subgroup discovery for continuous target concepts. In: Proceedings of ISMIS '09, Prague, pp 35-44

Aumann Y, Lindell Y (1999) A statistical theory for quantitative association rules. In: Proceedings of KDD'99, San Diego, pp 261-270

Bailey J, Dong G (2007) Contrast data mining: methods and applications. Tutorial at the IEEE international conference on data mining (ICDM), Omaha

Bay S, Pazzani M (2001) Detecting group differences: mining contrast sets. Data Min Knowl Discov 5(3):213-246

Bringmann B, Zimmermann A (2007) The chosen few: on identifying valuable patterns. In: Proceedings of the ICDM'07, Omaha, pp 63-72

Clark P, Boswell R (1991) Rule induction with CN2: some recent improvements. In: Proceedings of the European working session on learning (EWSL-91), Porto, pp 151-163

Clark P, Niblett T (1989) The CN2 induction algorithm. Mach Learn 3:261-283

Cover T, Thomas J (2006) Elements of information theory, 2nd ed. Wiley, New York

Daly O, Taniar D (2005) Exception rules in data mining. In: Encyclopedia of information science and technology (II), pp 1144-1148

Dong G, Zhang X, Wong L, Li J (1999) CAEP: classification by aggregating emerging patterns. In: Proceedings of DS'99, Tokyo, pp 30-42

Duivesteijn W, Knobbe A, Feelders A, van Leeuwen M (2010) Subgroup discovery meets bayesian networks: an exceptional model mining approach. In: Proceedings of the ICDM'10, Sydney, pp 158-167

Friedman J, Fisher N (1999) Bump hunting in high-dimensional data. Stat Comput 9(2):123-143

Garriga G, Kralj P, Lavrac N (2008) Closed sets for labeled data. J Mach Learn Res 9:559-580

Grosskreutz H, Paurat D (2011) Fast and memory-efficient discovery of the top-k relevant subgroups in a reduced candidate space. In: Proceedings of the ECML/PKDD '11, Athens, pp 533-548

Grosskreutz H, Rüping S (2009) On subgroup discovery in numerical domains. Data Min Knowl Discov $19(2): 210-226$

Grosskreutz H, Rüping S, Wrobel S (2008) Tight optimistic estimates for fast subgroup discovery. In: Proceedings of the ECML/PKDD'08, Antwerp, pp 440-456

Grosskreutz H, Boley M, Krause-Traudes M (2010) Subgroup discovery for election analysis: a case study in descriptive data mining. In: Proceedings of DS'10, no. 6332 in LNAI. Springer, New York, pp 57-71

Grünwald $\mathrm{P}$ (2007) The minimum description length principle. MIT Press, Cambridge

Han J, Cheng H, Xin D, Yan X (2007) Frequent pattern mining: current status and future directions. Data Min Knowl Discov 15(1):55-86

Heikinheimo H, Fortelius M, Eronen J, Mannila H (2007) Biogeography of european land mammals shows environmentally distinct and spatially coherent clusters. J Biogeogr 34(6):1053-1064

Klösgen W (1996) Advances in knowledge discovery and data mining, chap Explora: a multipattern and multistrategy discovery assistant. MIT Press, Cambridge, pp 249-271

Klösgen W (2002) Handbook of data mining and knowledge discovery, chap Subgroup discovery. Oxford University Press, Oxford

Knobbe A (2006) Multi-relational data mining. IOS Press, Amsterdam

Knobbe A, Ho E (2006a) Maximally informative $k$-itemsets and their efficient discovery. In: Proceedings of the KDD'06, Philadelphia, Berlin, pp 237-244

Knobbe A, Ho E (2006b) Pattern teams. In: Proceedings of the ECML PKDD’06, Berlin, pp 577-584

Knobbe A, Valkonet J (2009) Building classifiers from pattern teams. In: Proceedings of the ECML PKDD'09 workshop LeGo 2009, Bled, pp 77-93

Kocev D, Struyf J, Dzeroski S (2007) Beam search induction and similarity constraints for predictive clustering trees. In: LNCS KDID 2006, Berlin, pp 134-151

Kralj Novak P, Lavrač N, Webb G (2009) Supervised descriptive rule discovery: a unifying survey of contrast set, emerging pattern and subgroup mining. J Mach Learn Res 10:377-403

Kullback S, Leibler R (1951) On information and sufficiency. Ann Math Stat 22(1):79-86

Lavrač N, Kavšek B, Flach P, Todorovski L (2004) Subgroup discovery with CN2-SD. J Mach Learn Res 5:153-188 
Leman D, Feelders A, Knobbe A (2008) Exceptional model mining. In: Proceedings of the ECML/PKDD'08, vol 2, Antwerp, pp 1-16

Lemmerich F, Puppe F (2011) Local models for expectation-driven subgroup discovery. In: Proceedings of the ICDM'11, Vancouver

Lemmerich F, Rohlfs M, Atzmüller M (2010) Fast discovery of relevant subgroup patterns. In: Proceedings of FLAIRS, Daytona Beach

Liu B, Hsu W, Ma Y (2001) Discovering the set of fundamental rule changes. In: Proceedings of KDD'01, San Francisco, pp 335-340

Lowerre B (1976) The harpy speech recognition system. PhD thesis

Mannila H, Toivonen H (1996) Multiple uses of frequent sets and condensed representations. In: Proceedings of the KDD'96, Portland, pp 189-194

Mitchell-Jones A, Amori G, Bogdanowicz W, Krystufek B, Reijnders P, Spitzenberger F, Stubbe M, Thissen J, Vohralik V, Zima J (1999) The atlas of European mammals. Academic Press, London

Morishita S, Sese J (2000) Traversing itemset lattice with statistical metric pruning. In: Proceedings PODS, Dallas, pp 226-236

Nijssen S, Guns T, De Raedt L (2009) Correlated itemset mining in roc space: a constraint programming approach. In: Proceedings KDD’09, Paris, pp 647-656

Pasquier N, Bastide Y, Taouil R, Lakhal L (1999) Discovering frequent closed itemsets for association rules. In: Proceedings of the ICDT'99, Jerusalem, pp 398-416

Peng H, Long F, Ding C (2005) Feature selection based on mutual information: Criteria of max-dependency, max-relevance, and min-redundancy. IEEE Trans Pattern Anal Mach Intell 27(8):1226-1238

Pieters B, Knobbe A, Dzeroski S (2010) Subgroup discovery in ranked data, with an application to gene set enrichment. In: Proceedings preference learning workshop (PL 2010) at ECML PKDD '10, Barcelona

Shell P, Rubio JH, Barro GQ (1994) Improving search through diversity. In: AAAI, Seattle, pp 1323-1328

Tsoumakas G, Vilcek J, Spyromitros L (2010) MULAN: a java library for multi-label learning. http://mulan. sourceforge.net/

van Leeuwen M (2010) Maximal exceptions with minimal descriptions. Data Min Knowl Discov 21(2):259-276

van Leeuwen M, Knobbe A (2011) Non-redundant subgroup discovery in large and complex data. In: Proceedings of the ECML PKDD'11, Bled, pp 459-474

Vreeken J, van Leeuwen M, Siebes A (2011) Krimp: mining itemsets that compress. Data Min Knowl Discov 23(1):169-214

Webb G (1995) Opus: an efficient admissible algorithm for unordered search. J Artif Intell Res 3:431-465

Webb G (2001) Discovering associations with numeric variables. In: Proceedings of KDD’01, San Francisco, pp 383-388

Webb G, Butler S, Newlands D (2003) On detecting differences between groups. In: Proceedings of KDD'03, Washington, pp 256-265

Wrobel S (1997) An algorithm for multi-relational discovery of subgroups. In: Proceedings of PKDD 1997. Springer, Heidelberg, pp 78-87

Yan X, Han J (2002) gSpan: Graph-based substructure pattern mining. In: Proceedings of the ICDM'02, Maebashi, pp 721-724 